\title{
The Role of Nonequilibrium Thermo-Mechanical Statistics in Modern Technologies and Industrial Processes: An Overview
}

\author{
Clóves G. Rodrigues ${ }^{1}$, Antônio A. P. Silva ${ }^{2}$, Carlos A. B. Silva ${ }^{3}$, Áurea R. Vasconcellos ${ }^{4}$, J. Galvão Ramos ${ }^{4}$, Roberto Luzzi ${ }^{4}$ \\ ${ }^{1}$ Departamento de Física, Pontifícia Universidade Católica de Goias, 74605-010 Goiannia, Goias, Brazil \\ ${ }^{2}$ Departamento de Ciências Exatas, Universidade Federal de Lavras 37200-000 Lavras, MG, Brazil \\ ${ }^{3}$ Instituto Tecnológico de Aeronáutica, 12228-901 São José dos Campos, SP, Brazil \\ ${ }^{4}$ Condensed Matter Physics Department, Institute of Physics "Gleb Wataghin”, \\ University of Campinas-Unicamp, 13083-970 Campinas, SP, Brazil
}

(Received on 27 November, 2009)

\begin{abstract}
The nowadays notable development of all the modern technology, fundamental for the progress and well being of world society, imposes a great deal of stress in the realm of basic Physics, more precisely on ThermoStatistics. We do face situations in electronics and optoelectronics involving physical-chemical systems farremoved-from equilibrium, where ultrafast (in pico- and femto-second scale) and non-linear processes are present. Further, we need to be aware of the rapid unfolding of nano-technologies and use of low-dimensional systems (e.g., nanometric quantum wells and quantum dots in semiconductor heterostructures). All together this demands having an access to a Statistical Mechanics being efficient to deal with such requirements. It is worth noticing that the renowned Ryogo Kubo once stated that "statistical mechanics has been considered a theoretical endeavor. However, statistical mechanics exists for the sake of the real world, not for fictions. Further progress can only be hoped by close cooperation with experiment". Moreover, one needs to face the study of soft matter and fluids with complex structures (usually of the average self-affine fractal-like type). This is relevant for technological improvement in industries like, for example, that of polymers, petroleum, cosmetics, food, electronics and photonics (conducting polymers and glasses), in medical engineering, etc. It is then required to introduce a thermo-hydrodynamics going well beyond the classical (Onsagerian) one. Moreover, in the both type of situations above mentioned there often appear difficulties of description and objectivity (existence of so-called "hidden constraints"), which impair the proper application of the conventional ensemble approach used in the general, logically and physically sound, and well established Boltzmann-Gibbs statistics. A tentative to partially overcome such difficulties consists in resorting to non-conventional approaches. Here we briefly describe the construction of a Non-Equilibrium Statistical Ensemble Formalism (NESEF) that can deal, within a certain degree of success, with the situations above described. Several particular instances involving experimental observations and measurements in the area of semiconductor physics and in physics of fluids, which were analyzed in the context of the theory, are summarized. They comprise the cases of ultrafast optical spectroscopy; optical and transport processes in low-dimensional complex semiconductors; nonlinear transport in doped highly-polar semiconductors (of use in "blue diodes") under moderate to high electric fields; nonlinear higher-order thermo-hydrodynamics in fluids under driven flow, in normal solutions and in complex situations as in solutions of polymers, micelles, DNA, and in microbatteries.
\end{abstract}

Keywords: statistical physics; irreversible thermodynamics; science and society

\section{INTRODUCTION}

It can be noticed that at the present moment, in what some authors call the "Third Industrial Revolution", or others argue that it is a large evolution of the "Second Industrial Revolution", the notable development of the so-called "first point technologies" (or "advanced modern technologies") and the accompanying intense R\&D (research and development), ask for Physics to bring to the forefront the Physics of Systems far-away-from Equilibrium [1-4] (the condition of functioning of many systems), and with it the Physics of Non-Linear Processes [5-7]. Nonlinearity (in the kinetic equations providing the evolution laws of the macroscopic state of the system) comes to have a relevant role, and with it enters into play the disciplines of Synergetic and Self-Organization [8,9] associated to systems with complex behavior (for short, complex systems); namely, formation of coherent structures organized at a macroscopic level (space, time, or functional organization), as it is the case of the omnipresent laser or life in the biosphere [10,11]. Finally, it needs be also considered the Mesoscopic Physics [12-16], necessary to deal with processes in nanometric scale in space and femto-second scale in time [17-22], the case of the so-called fractal structures [23], the emerging area of soft matter [24], and so on.

Thus, the nowadays notable development of all the modern technologies, fundamental for the progress and well being of world society, posses, a great deal of stress in the realm of basic Physics, more precisely on Thermo-Statistics. We do face situations in electronics and optoelectronics involving physical-chemical systems far-removed-from equilibrium, where ultrafast (in pico- and femto-second scale) and non-linear processes are present. Further, we need to be aware of the rapid unfolding of nano-technologies and use of low-dimensional systems (e.g., nanometric quantum wells and quantum dots in semiconductors heterostructures). All together this demands having an access to a Statistical Mechanics being efficient to deal with such requirements. Moreover, one needs to face the study of soft matter and fluids with complex structures (usually of the average selfaffine fractal-like type). This is relevant for technological improvement in industries like, for example, that of polymers, petroleum, cosmetics, food, electronics and photonics (conducting polymers and glasses), in medical engineering, etc. It is then required to introduce a thermo-hydrodynamics going well beyond the classical (Onsagerian) one. In the both type of situations above mentioned there often appear difficulties of description and objectivity (existence of so-called 
"hidden constraints"), which impair the proper application of the conventional ensemble approach used in the general, logically and physically sound, and well established BoltzmannGibbs statistics. A tentative to partially overcome such difficulties consists in resorting to non-conventional approaches. In addition, the present technological moment, and commercial interest, claims for miniaturization of devices, and then this rises the question if our comprehension of the physics of devices and their functioning can be extrapolated to the ultrasmall and ultra-short scales, in space and time respectively. It is Statistical Mechanics (or Thermo-Statistics) that provides the basic scientific foundations to the above questions, more precisely, Nonequilibrium Statistical Mechanics whose evolution we describe in next Section. It is followed by a brief description of the construction of a Non-Equilibrium Statistical Ensemble Formalism (NESEF) that can deal with the situations above mentioned. Particularly, it has been applied with success in the cases of ultrafast optical spectroscopy; optical and transport processes in low-dimensional complex semiconductors; nonlinear transport in doped highly-polar semiconductors (of use in "blue Diodes") under moderate to high electric fields; nonlinear higher-order thermo-hydrodynamics in fluids under driven flow, in normal solutions and in complex situations as in solutions of polymers, micelles, DNA, and in microbatteries.

\section{NONEQUILIBRIUM STATISTICAL MECHANICS}

Back in 1979 Oliver Penrose [25] stated that Statistical Mechanics is notorious for conceptual problems to which it is difficult to give a convincing answer. This notwithstanding, Statistical Mechanics for systems in equilibrium provides - via Gibb's ensemble algorithm - an extraordinarily precise description of large physical systems. When a quite few experimental measurements (which, it is recalled, are of a macroscopic character) are available to define the state of the system, generally information associated to constants of motion, the description advocated by Gibbs provides results that are impressive. Nowadays the development of sophisticated mathematical techniques allow us to calculate in detail the contribution of the interactions between the particles to the physical properties of the system. In this way the essential characteristics of the systems in equilibrium are well understood. However, the situation is at present not so glossy in the case of systems far-away-from equilibrium.

Let us recall that what Gibbs called, for the first time, Statistical Mechanics [26] is a careful and brilliant theoretical construction of a large scope that superseded the kinetic theory of the nineteenth century. Gibbs's theory looks, on the one side, for an acceptable and fundamental foundation, on microscopic basis, of phenomenological thermodynamics in its both aspects, namely, the physical and the conceptual. However, on the other side, it went beyond that, trying to describe all the macroscopic physical properties of matter from a microscopic level by also providing foundations to response function theory. Moreover, related to this, there is in a sense another objective that can be denominated as the inverse problem, that is, to built microscopic models for the description of the underlying dynamics that may lead to the prediction of macroscopic observables, trying ways to test such models of microscopic interactions.

The construction of the truly successful Gibbs method is usually described in the textbooks on the subject in a scheme that we may call as orthodox: Basically, deterministic and reversible mechanics (based in the classical case on the notion of well defined trajectories in phase space and integrability, and in the quantum case on the state evolution governed by Schrödinger equation) is accompanied with a component borrowed from probability theory together with ad hoc hypotheses as the principle of macroscopic reproducibility and that of equal a priori probabilities for the isolated system, in order to built the basic ensemble known as the microcanonical one, from which the others are derived.

Probability theory appears to be an "inexorable" necessity in any construction with which we may be trying to describe phenomena at the macroscopic level. Richard Feynman has stated [27] that, it is not our ignorance of the internal gears, of the internal complications, that makes nature to have probability in it. It seems to be somehow intrinsic; Nature herself does not even know which way a system is going to go. Later on Jacob Bronowski advanced the idea that [28]

"this is the revolutionary thought in modern science. It replaces the concept of the inevitable effect by that of the probable trend. Its technique is to separate so far as possible the steady trend from local fluctuations. The future does not already exist; it can only be predicted".

Bronowski and others have emphasized the concept of probability as seemingly fundamental to the development of the science thought. And this seems to go well beyond the field of the natural sciences, including also the sociocultural ones, because it appears to be a concept fundamental to the scientific study of any type of dynamic systems, let it be physical, chemical, biological, ecological, economic, historical, archeological, social, etc. As a political scientist put it, we are evolving towards a world that is more complex, with more freedom, more diversity, with more possibilities of change, and, consequently, less stability. In fact, again following Bronowski: "The different branches of science may seem so far apart only because we lack the common method on which they grow and which holds them together organically [...] The statistical concept of chance may come as dramatically to unify the scattered pieces of science future [...] We are on the threshold of another scientific revolution. The concept of natural law is changing”. [28]

It is generally considered that the aim of Statistical Mechanics of many-body systems away from equilibrium is to determine their thermodynamic properties, and the evolution in time of their macroscopic observables, in terms of the dynamical laws which govern the motion of their constitutive elements. This implies, first, in the construction of an irreversible thermodynamics and a thermo-hydrodynamics (the latter meaning the particle and energy motion in fluids, rheological properties, etc., with the transport coefficients depending on the macroscopic thermodynamic state of the system). Second, we need to face the all-important derivation of a generalized nonlinear quantum kinetic theory and a response function theory, which are of fundamental relevance to connect theory with observation and experiment, basic for the corroboration of any theory (e.g. Ref. [29]), that is, the aspect of synthesis in the scientific method born in the seventeenth century.

As stated at the beginning of this section, Oliver Penrose 
[25] has noted that Statistical Mechanics is notorious for conceptual problems to which is difficult to give a convincing answer, which are, mainly:

- What is the physical significance of a Gibbs' ensemble?

- How can we justify the standard ensembles used in equilibrium theory?

- What are the right ensembles for nonequilibrium?

- How can we reconcile the reversibility of microscopic mechanics with the irreversibility of macroscopic behavior?

Moreover, related to the case of many-body systems out of equilibrium, Ryogo Kubo, in the opening address in the Oji Seminar [30], told us that statistical mechanics of nonlinear phenomena is just in its infancy and further progress can only be hoped by closed cooperation with experiment. Some progress has been achieved since then, and we try in this paper to describe some attempts in the direction to provide a path for one particular initial programme to face the questions posited above.

In the study of the macroscopic state of nonequilibrium systems we face greater difficulties than those in the theory of equilibrium systems. This is mainly due to the fact that a more detailed analysis is necessary to determine the temporal dependence of measurable properties, and to calculate transport coefficients which are time-dependent (that is, depending on the evolution in time of the nonequilibrium macrostate of systems where dissipative processes are unfolding), and which are also space dependent. That dependence is nonlocal in space and non-instantaneous in time, as it encompasses space and time correlations. Robert Zwanzig [2] has summarized the basic goals of nonequilibrium statistical mechanics as consisting of:

1. To derive transport equations and to grasp their structure;

2. To understand how the approach to equilibrium occurs in natural systems;

\section{To study the properties of the steady state; and}

4. To calculate the instantaneous values and temporal evolution of the physical quantities which specify the macroscopic state of the system.

Also according to Zwanzig, for the purpose to face these questions there exist several approaches which can be classified as:

(a) Intuitive techniques;

(b) Techniques based on the generalization of the kinetic theory of gases;

(c) Techniques based on the theory of stochastic processes;

(d) Expansions from an initial equilibrium ensemble;

(e) Generalizations of Gibbs' ensemble formalism;

and nowadays we should also include

\section{(f) Computational Modelling Methods.}

The last two are presently the most favorable approaches for providing very satisfactory methods for dealing with nonequilibrium system in general situations.

The Nonequilibrium Molecular Dynamics (NMD) is a computational method created for modelling physical systems at the microscopic level, in time and distance, being a good technique to study the molecular behavior of several physical processes [31]. Together with the so-called Monte Carlo method are part of what is know as Numeric Simulation Methods (e.g. [32]), or Computational Physics (which is to be added to the old classification of Experimental and Theoretical Physics) [33].

NMD is a method used for the study of properties of matter in which the direct integration of the dynamic equations of motion of the many-body system is done. The first numerical simulation is due to Alder and Wainwright [34], done for a system of hard spheres. Years later Rahman solved the case of molecules interacting through a Lenard-Jones potential [35]. Later on Car and Parrinello largely improved the approach in what is dubbed as ab initio molecular dynamics [36,37]. Here classical and quantum dynamics are coupled in such a way that it is possible to extend the simulation to systems which are of difficult modelation.

The other approach, which we favor here, is the one of item (e) above, i.e. connected with Penrose's question stated previously concerning if there are, and which are, right ensembles for nonequilibrium problems. In the absence of a Gibbs-style ensembles approach, for a long time different kinetic theories were used, with variable success, to deal with the great variety of nonequilibrium phenomena occurring in physical systems. An approach for constructing a Nonequilibrium Statistical Ensemble Formalism (NESEF for short), which appears to provide grounds for a general prescription to choose appropriate ensembles for nonequilibrium systems, is briefly described in next Section. The formalism has an accompanying nonlinear quantum transport theory of a large scope (which encompasses as particular limiting cases Boltzmann's and Mori's approaches and in its general form provides far-reaching generalization of them), a response function theory for arbitrarily-away-from equilibrium systems, a statistical thermodynamics (the so-called Informational Statistical Thermodynamics), and an accompanying higher-order thermo-hydrodynamics.

NESEF appears as a very powerful, concise, based on sound principles, and elegant formalism of a broad scope to deal with systems arbitrarily away from equilibrium. Zwanzig sated that the formalism "has by far the most appealing structure, and may yet become the most effective method for dealing with nonlinear transport processes" [2].

Later developments have confirmed Zwanzig's prediction. The present structure of the formalism consists in a vast extension and generalization of earlier pioneering approaches, among which we can pinpoint the works of Kirkwood [38], Green [39], Mori-Oppenheim-Ross [40], Mori [41], and Zwanzig [42]. NESEF has been approached from different points of view: some are based on heuristic arguments, others on projection-operator techniques (the former following Kirkwood and Green and the latter following Zwanzig and Mori). 
The formalism has been particularly systematized and largely improved by the Russian School of statistical physics, which can be considered to have been initiated by the renowned Nicolai Nicolaievich Bogoliubov (e.g. see ref. [43]) and we may also name Nicolai Sergeievich Krylov [44], and more recently mainly through the relevant contributions by Dimitrii Zubarev [1,45], Sergei Peletminskii [46,47], and others. We present in Refs. [3,4,48,49] a systematization, as well as generalizations and conceptual discussions of the matter.

It may be noticed that these different approaches to NESEF can be brought together under a unique variational principle. This has been originally done by Zubarev and Kalashnikov [50], and later on reconsidered in Refs. [3,45]. It consists on the maximization, in the context of Information Theory, of Gibbs statistical entropy (that is, the average of minus the logarithm of the statistical distribution function, which in Communication Theory is Shannon informational entropy [51-53]) subjected to certain constraints and including non-locality in space, retro-effects, and irreversibility on the macroscopic level.

Let us briefly describe its foundations and construction (for a complete in depth presentation see Refs. $[3,4])$.

\section{BRIEF REVIEW OF NESEF}

A brief review of the foundations of the Non-Equilibrium Statistical Ensemble Formalism (NESEF) [1,3,4,40,45-47] is presented. For such purpose first it needs be noticed that for systems away from equilibrium several important points need be carefully taken into account in each case under consideration:

1. The choice of the basic variables (a wholly different choice than in equilibrium when it suffices to take a set of those which are constants of motion), which is to be based on an analysis of what sort of macroscopic measurements and processes are actually possible, and moreover, one is to focus attention not only on what can be observed but also on the character and expectative concerning the equations of evolution for these variables (e.g. Refs. [2-4]). We also notice that even though at the initial stage we would need to introduce all the observables of the system, and eventually variances, as time elapses more and more contracted descriptions can be used when it enters into play Bogoliubov's principle of correlation weakening and the accompanying hierarchy of relaxation times [54].

2. The question of irreversibility (or Eddington's arrow of time) on what Rudolf Peierls stated that: "In any theoretical treatment of transport problems, it is important to realize at what point the irreversibility has been incorporated. If it has not been incorporated, the treatment is wrong. A description of the situation that preserves the reversibility in time is bound to give the answer zero or infinity for any conductivity. If we do not see clearly where the irreversibility is introduced, we do not clearly understand what we are doing" [55].
3. Historicity needs be introduced, that is, the idea that it must be incorporated all the past dynamics of the system (or historicity effects), all along the time interval going from a starting description of the macro-state of the sample in the given experiment, say at $t_{0}$, up to the time $t$ when a measurement is performed. This is a quite important point in the case of dissipative systems as emphasized among others by John Kirkwood, Melvin Green, Robert Zwanzig and Hazime Mori [3842].

Concerning the question of the choice of the basic variables, differently to the case in equilibrium, immediately after the open system of $N$ particles, in contact with external sources and reservoirs, has been driven out of equilibrium it would be necessary to describe its state in terms of all its observables and eventually, introducing direct and crosscorrelation fluctuations. But, this is equivalent to have access to the so-called one-particle (or single-particle), $\hat{n}_{1}$, and twoparticle, $\hat{n}_{2}$, dynamical operators.

This is so because, we recall, all observable quantities and their variances can be expressed, at the microscopic mechanical level, in terms of these dynamical operators. For a description of mechanical states by means of these reduced density operators we refer to the already classical paper by Ugo Fano [56].

For the sake of completeness, we notice that in classical mechanics the one particle and two-particle operators, $\hat{n}_{1}$ and $\hat{n}_{2}$ are given, respectively, by

$$
\hat{n}_{1}(\mathbf{r}, \mathbf{p})=\sum_{j=1}^{N} \delta\left(\mathbf{r}-\mathbf{r}_{j}\right) \delta\left(\mathbf{p}-\mathbf{p}_{j}\right)
$$

$\hat{n}_{2}\left(\mathbf{r}, \mathbf{p}, \mathbf{r}^{\prime}, \mathbf{p}^{\prime}\right)=\sum_{j \neq k=1}^{N} \delta\left(\mathbf{r}-\mathbf{r}_{j}\right) \boldsymbol{\delta}\left(\mathbf{p}-\mathbf{p}_{j}\right) \delta\left(\mathbf{r}^{\prime}-\mathbf{r}_{k}\right) \boldsymbol{\delta}\left(\mathbf{p}^{\prime}-\mathbf{p}_{k}\right)$,

where $\mathbf{r}_{j}$ and $\mathbf{p}_{j}$ are the coordinate and linear momentum of the $j$-th particle in phase space and $\mathbf{r}, \mathbf{p}$, etc., the continuous values of position and momentum, which are sometimes called field variables (for simplicity we take the case of a single class of particles; otherwise we must write dynamical operators for each kind of particle).

In quantum mechanics the one- and two-particle density operators are ( $\sigma$ is the spin index)

$$
\begin{aligned}
& \hat{n}_{1}\left(\mathbf{r}, \sigma, \mathbf{r}^{\prime}, \sigma^{\prime}\right)=\psi_{\sigma}^{\dagger}(\mathbf{r}) \psi_{\sigma^{\prime}}\left(\mathbf{r}^{\prime}\right), \\
& \hat{n}_{2}\left(\mathbf{r}_{1}, \sigma_{1}, \mathbf{r}_{2}, \sigma_{2}, \mathbf{r}_{2}^{\prime}, \sigma_{2}^{\prime}, \mathbf{r}_{1}^{\prime}, \sigma_{1}^{\prime}\right)=\psi_{\sigma_{1}}^{\dagger}\left(\mathbf{r}_{1}\right) \psi_{\sigma_{2}}^{\dagger}\left(\mathbf{r}_{2}\right) \times \\
& \psi_{\sigma_{2}^{\prime}}\left(\mathbf{r}_{2}^{\prime}\right) \psi_{\sigma_{1}^{\prime}}\left(\mathbf{r}_{1}^{\prime}\right)
\end{aligned}
$$

where $\psi\left(\psi^{\dagger}\right)$ are single-particle field operators in second quantization (an excellent didactic description of them is available in the article by B. Robertson of Ref. [57]).

Hence, on the one hand, the non-equilibrium statistical operator $\mathfrak{R}_{\varepsilon}(t)$ is dependent on these quantities, and, on the other hand, the macro-variables for describing the nonequilibrium thermodynamic state of the system are the average value of the same quantities over the non-equilibrium ensemble, i.e.

$$
f_{1}(\mathbf{r}, \mathbf{p} ; t)=\operatorname{Tr}\left\{\hat{n}_{1}(\mathbf{r}, \mathbf{p}) \Re_{\varepsilon}(t)\right\},
$$




$$
f_{2}\left(\mathbf{r}, \mathbf{p}, \mathbf{r}^{\prime}, \mathbf{p}^{\prime} ; t\right)=\operatorname{Tr}\left\{\hat{n}_{2}\left(\mathbf{r}, \mathbf{p}, \mathbf{r}^{\prime}, \mathbf{p}^{\prime}\right) \Re_{\varepsilon}(t)\right\},
$$

in classical mechanics; $f_{1}(\mathbf{r}, \mathbf{p} ; t)$ has the role of a generalized Boltzmann distribution function and $T r$, we recall, stands for performing the trace operation (integration over phase space in classical mechanics).

On the question of irreversibility Nicolai S. Krylov [44] considered that there always exists a physical interaction between the measured system and the external world that is constantly "jolting" the system out of its exact microstate. Thus, the instability of trajectories and the unavoidable finite interaction with the outside would guarantee the working of a "crudely prepared" macroscopic description. In the absence of a proper way to introduce such effect, one needs to resort to the so-called interventionist's approach, which is grounded on the basis of such ineluctable process of randomization leading to the asymmetric evolution of the macro-state.

The "intervention" consists into introducing in the Liouville equation of the statistical operator of the otherwise isolated system, a particular source accounting for Krylov's "jolting" effect, in the form (written for the logarithm of the statistical operator)

$$
\frac{\partial}{\partial t} \ln \Re_{\varepsilon}(t)+\frac{1}{i \hbar}\left[\ln \Re_{\varepsilon}(t), \hat{H}\right]=-\varepsilon\left[\ln \Re_{\varepsilon}(t)-\ln \bar{\Re}_{\varepsilon}(t, 0)\right],
$$

where $\varepsilon$ (kind of reciprocal of a relaxation time) is taken to go +0 after the calculations of average values has been performed. Such mathematically inhomogeneous term, in the otherwise normal Liouville equation, implies in a continuous tendency of relaxation of the statistical operators towards a referential one, $\overline{\mathfrak{R}}$, which, as discussed below, represents an instantaneous quasi-equilibrium condition.

We can see that Eq. (7) consists of a regular Liouville equation but with an infinitesimal source, which provides Bogoliubov's symmetry breaking of time reversal and is responsible for disregarding the advanced solutions $[1,3,58]$. This is described by a Poisson distribution and the result at time $t$ is obtained by averaging over all $t^{\prime}$ in the interval $\left(t_{0}, t\right)$, once the solution of Eq. (7) is

$$
\Re_{\varepsilon}(t)=\exp \left\{-\hat{S}(t, 0)+\int_{t_{0}}^{t} d t^{\prime} e^{\varepsilon\left(t^{\prime}-t\right)} \frac{d}{d t^{\prime}} \hat{S}\left(t^{\prime}, t^{\prime}-t\right)\right\},
$$

where

$$
\begin{gathered}
\hat{S}(t, 0)=-\ln \bar{\Re}(t, 0), \\
\hat{S}\left(t^{\prime}, t^{\prime}-t\right)=\exp \left\{-\frac{1}{i \hbar}\left(t^{\prime}-t\right) \hat{H}\right\} \hat{S}\left(t^{\prime}, 0\right) \times \\
\exp \left\{\frac{1}{i \hbar}\left(t^{\prime}-t\right) \hat{H}\right\},
\end{gathered}
$$

and the initial condition at time $t_{0}$, when the formalism begins to be applied, is

$$
\Re_{\varepsilon}\left(t_{0}\right)=\bar{\Re}\left(t_{0}, 0\right) .
$$

This time $t_{0}$, of initiation of the statistical description, is usually taken in the remote past $\left(t_{0} \longrightarrow-\infty\right)$ introducing an adiabatic switch on of the relaxation processes, and in Eq. (8) the integration in time in the interval $\left(t_{0}, t\right)$ is weighted by the kernel $\exp \varepsilon\left(t^{\prime}-t\right)$. The presence of this kernel introduces a kind of evanescent history as the system macro-state evolves toward the future from the boundary condition of Eq. (11) at time $\left(t_{0} \longrightarrow-\infty\right)$ (a result of the presence of the exponential in Eq. (8) which accounts for the dissipative evolution of the state of the system, a fact evidenced in the resulting kinetic theory $[1,3,4,40,45,47,57,59]$ which clearly indicates that a fading memory process has been introduced). Moreover, in most cases we can consider the system as composed of the system of interest (on which we are performing an experiment) in contact with ideal reservoirs. Thus, we can write

$$
\Re_{\varepsilon}(t)=\rho_{\varepsilon}(t) \times \rho_{R},
$$

where $\rho_{\varepsilon}(t)$ is the statistical operator of the nonequilibrium system and $\rho_{R}$ the stationary one of the ideal reservoirs, with $\rho_{\varepsilon}(t)$ given then by

$$
\rho_{\varepsilon}(t)=\exp \left\{-\hat{S}(t, 0)+\int_{-\infty}^{t} d t^{\prime} e^{\varepsilon\left(t^{\prime}-t\right)} \frac{d}{d t^{\prime}} \hat{S}\left(t^{\prime}, t^{\prime}-t\right)\right\},
$$

having the initial value $\bar{\rho}\left(t_{0}, 0\right)\left(t_{0} \longrightarrow-\infty\right)$, and where

$$
\begin{aligned}
& \hat{S}(t, 0)=-\ln \bar{\rho}(t, 0), \\
\hat{S}\left(t^{\prime}, t^{\prime}-t\right)= & \exp \left\{-\frac{1}{i \hbar}\left(t^{\prime}-t\right) \hat{H}\right\} \ln \bar{\rho}\left(t^{\prime}, 0\right) \times \\
& \exp \left\{\frac{1}{i \hbar}\left(t^{\prime}-t\right) \hat{H}\right\} .
\end{aligned}
$$

Finally, it needs be provided the auxiliary statistical operator $\bar{\rho}(t, 0)$. It defines an instantaneous distribution at time $t$, which describes a "frozen" equilibrium which at such given time defines the macroscopic state of the system, and for that reason is sometimes dubbed as the quasi-equilibrium statistical operator. On the basis of this (or, alternatively, via the extremum-principle procedure [3,60-65], and considering the description of the non-equilibrium state of the system in terms of the single- and two-particle density operators, the reference or instantaneous quasi-equilibrium statistical operator is taken as a canonical-like one given by

$$
\begin{aligned}
\bar{\rho}(t, 0)= & \exp \left\{-\phi(t)-\int d^{3} r \int d^{3} p F_{1}(\mathbf{r}, \mathbf{p} ; t) \hat{n}_{1}(\mathbf{r}, \mathbf{p})-\right. \\
& -\int d^{3} r \int d^{3} p \int d^{3} r^{\prime} \int d^{3} p^{\prime} \times \\
& \left.F_{2}\left(\mathbf{r}, \mathbf{p} ; \mathbf{r}^{\prime}, \mathbf{p}^{\prime} ; t\right) \hat{n}_{2}\left(\mathbf{r}, \mathbf{p}, \mathbf{r}^{\prime}, \mathbf{p}^{\prime}\right)\right\}
\end{aligned}
$$

in the classical case, with $\phi(t)$ ensuring the normalization of $\bar{\rho}$, and playing the role of a kind of a logarithm of a partition function, say, $\phi(t)=\ln \bar{Z}(t)$. Moreover, in this Eq. (16), $F_{1}$ and $F_{2}$, are the non-equilibrium thermodynamic variables associated to each kind of basic dynamical variables, $\hat{n}_{1}$ and $\hat{n}_{2}$ respectively (Lagrange multipliers in the extremum-principle approach).

An alternative equivalent and complete description, highly convenient for deriving a kinetic theory of hydrodynamic (a nonlinear higher-order one), consists in the construction of a generalized nonequilibrium grand-canonical distribution. Considering for simplicity the case of retaining only 
$\hat{n}_{1}$ as basic dynamical variable, this follows by redefining the nonequilibrium thermodynamic variable $F_{1}$ in the form

$$
\begin{aligned}
F_{1}(\mathbf{r}, \mathbf{p} ; t)= & F_{n}(\mathbf{r} ; t)+\frac{\mathbf{p}}{m} \cdot \mathbf{F}_{n}(\mathbf{r} ; t)+\frac{p^{2}}{2 m} F_{h}(\mathbf{r} ; t)+ \\
& +\frac{p^{2}}{2 m} \frac{\mathbf{p}}{m} \cdot \mathbf{F}_{h}(\mathbf{r} ; t)+ \\
& +\sum_{r \geq 2}\left[F_{h}^{[r]}(\mathbf{r}, t) \otimes \frac{p^{2}}{2 m} u^{[r]}(\mathbf{p})+\right. \\
& \left.+F_{n}^{[r]}(\mathbf{r}, t) \otimes u^{[r]}(\mathbf{p})\right],
\end{aligned}
$$

where

$$
u^{[r]}(\mathbf{p})=\left[\frac{\mathbf{p}}{m} \cdots(r-\text { times }) \cdots \frac{\mathbf{p}}{m}\right],
$$

is a $r$-rank tensor consisting of the tensorial product of $r$-times the velocity $\mathbf{p} / m$, and $\otimes$ stands for fully contracted product of tensors, which when introduced in the statistical operator of Eq. (16), after disregarding the contribution $F_{2} \hat{n}_{2}$, it acquires the form

$$
\begin{aligned}
\bar{\rho}(t, 0)= & \exp \left\{-\phi(t)-\int d^{3} r\left[F_{h}(\mathbf{r}, t) \hat{h}(\mathbf{r})+\right.\right. \\
& +F_{n}(\mathbf{r}, t) \hat{n}(\mathbf{r})+\mathbf{F}_{h}(\mathbf{r}, t) \cdot \hat{\mathbf{I}}_{h}(\mathbf{r})+ \\
& +\mathbf{F}_{n}(\mathbf{r}, t) \cdot \hat{\mathbf{I}}_{n}(\mathbf{r})+\sum_{r \geq 2}\left[F_{h}^{[r]}(\mathbf{r}, t) \otimes \hat{I}_{h}^{[r]}(\mathbf{r})+\right. \\
& \left.\left.\left.+F_{n}^{[r]}(\mathbf{r}, t) \otimes \hat{I}_{n}^{[r]}(\mathbf{r})\right]\right]\right\}
\end{aligned}
$$

where

$$
\begin{gathered}
\hat{h}(\mathbf{r})=\int d^{3} p \frac{p^{2}}{2 m} \hat{n}_{1}(\mathbf{r}, \mathbf{p}) ; \hat{n}_{1}(\mathbf{r})=\int d^{3} p \hat{n}_{1}(\mathbf{r}, \mathbf{p}), \\
\hat{\mathbf{I}}_{h}(\mathbf{r})=\int d^{3} p \frac{\mathbf{p}}{m} \frac{p^{2}}{2 m} \hat{n}_{1}(\mathbf{r}, \mathbf{p}) ; \\
\hat{\mathbf{I}}_{n}(\mathbf{r})=\int d^{3} p \frac{\mathbf{p}}{m} \hat{n}_{1}(\mathbf{r}, \mathbf{p}), \\
\hat{I}_{h}^{[r]}(\mathbf{r})=\int d^{3} p u^{[r]}(\mathbf{p}) \frac{p^{2}}{2 m} \hat{n} \hat{n}_{1}(\mathbf{r}, \mathbf{p}) ; \\
\hat{I}_{n}^{[r]}(\mathbf{r})=\int d^{3} p u^{[r]}(\mathbf{p}) \hat{n}(\mathbf{r}, \mathbf{p}),
\end{gathered}
$$

which are the densities of energy, $\hat{h}$, and particles, $\hat{n}$, and their fluxes of all orders (the vectorial ones and the tensorial with $r \geq 2$ ). They have as the conjugated nonequilibrium thermodynamic variables set

$$
\begin{aligned}
& \left\{F_{h}(\mathbf{r}, t), F_{n}(\mathbf{r}, t), \mathbf{F}_{h}(\mathbf{r}, t), \mathbf{F}_{n}(\mathbf{r}, t),\left\{F_{h}^{[r]}(\mathbf{r}, t)\right\},\right. \\
& \left.\left\{F_{n}^{[r]}(\mathbf{r}, t)\right\}\right\},
\end{aligned}
$$

and it can be noticed that, alternatively, this set of variables completely describe the non-equilibrium thermodynamic state of the system. They are related to the basic set of macro-variables by the relations (which can be considered as the equations of state in arbitrary non-equilibrium conditions $[3,4,13-16])$

$$
\begin{aligned}
I_{h}^{[r]}(\mathbf{r}, t) & =\operatorname{Tr}\left\{\hat{\mathbf{I}}_{h}(\mathbf{r}) \bar{\rho}(t, 0)\right\}=-\frac{\delta \phi}{\delta F_{h}^{[r]}(\mathbf{r}, t)}= \\
& =-\frac{\delta \ln \bar{Z}(t)}{\delta F_{h}^{[r]}(\mathbf{r}, t)}, \\
I_{n}^{[r]}(\mathbf{r}, t) & =\operatorname{Tr}\left\{\hat{\mathbf{I}}_{n}(\mathbf{r}) \bar{\rho}(t, 0)\right\}=-\frac{\delta \phi}{\delta F_{n}^{[r]}(\mathbf{r}, t)}= \\
& =-\frac{\delta \ln \bar{Z}(t)}{\delta F_{n}^{[r]}(\mathbf{r}, t)},
\end{aligned}
$$

where $r=0$ for the densities, $r=1$ for vector (first order) fluxes, and $r \geq 2$ for the higher-order tensor fluxes, we have used that $\rho_{\varepsilon}$ and $\bar{\rho}$, at each time $t$, define the same average values for the basic variables only $[1,3]$, and $\delta$ stands for functional differential [66]. A complete description of the NESEF foundations for Irreversible Thermodynamics is available in Refs. [13-16].

\section{NESEF-BASED KINETIC THEORY}

The NESEF-based Nonlinear Kinetic Theory of relaxation process basically consists into taking the average over the nonequilibrium ensemble of Heisenberg (or Hamilton at the classical level) equations of motion of the dynamical operator for the observable, say, $A(\mathbf{r}, t)$ under consideration, i.e.

$$
\frac{d}{d t} A(\mathbf{r}, t)=\frac{d}{d t} \operatorname{Tr}\left\{\hat{A}(\mathbf{r}) \rho_{\varepsilon}(t)\right\}=\operatorname{Tr}\left\{\frac{1}{i \hbar}[\hat{A}(\mathbf{r}), \hat{H}] \rho_{\varepsilon}(t)\right\} .
$$

The practical handling of this NESEF-Kinetic Theory is described in Refs. [1,3,47,67-69] and mainly in [59].

Here we briefly noticed that the Markovian limit of the kinetic theory is of particular relevance as a result that, for a large class of problems, the interactions involved are weak and the use of this lowest order in the equations of motion constitutes an excellent approximation of good practical value. By means of a different approach, E. B. Davies [70] has shown that in fact the Markovian approach can be validated in the weak coupling (in the interaction) limit (retaining only the quadratic contribution). Essentially it implies in only retaining the interaction strengths up to second order.

Explicitly written, the Markovian equations in the kinetic theory for a set of quantities $\left\{A_{j}(\mathbf{r}, t)\right\}$ with $j=1,2, \ldots$ are

$$
\frac{\partial}{\partial t} A_{j}(\mathbf{r}, t)=J_{j}^{(0)}(\mathbf{r}, t)+J_{j}^{(1)}(\mathbf{r}, t)+J_{j}^{(2)}(\mathbf{r}, t),
$$

where

$$
\begin{aligned}
& J_{j}^{(0)}(\mathbf{r}, t)=\operatorname{Tr}\left\{\frac{1}{i \hbar}\left[\hat{A}_{j}(\mathbf{r}), \hat{H}_{0}\right] \bar{\rho}(t, 0) \times \rho_{R}\right\}, \\
& J_{j}^{(1)}(\mathbf{r}, t)=\operatorname{Tr}\left\{\frac{1}{i \hbar}\left[\hat{A}_{j}(\mathbf{r}), \hat{H}^{\prime}\right] \bar{\rho}(t, 0) \times \rho_{R}\right\},
\end{aligned}
$$




$$
\begin{aligned}
& \text { and } J_{j}^{(2)}(\mathbf{r}, t)={ }_{I} J_{j}^{(2)}(\mathbf{r}, t)+{ }_{I I} J_{j}^{(2)}(\mathbf{r}, t) \text {, with } \\
& { }_{I} J_{j}^{(2)}(\mathbf{r}, t)=\frac{1}{(i \hbar)^{2}} \int_{-\infty}^{t} d t^{\prime} e^{\varepsilon\left(t^{\prime}-t\right)} \times \\
& \operatorname{Tr}\left\{\left[\hat{H}^{\prime}\left(t^{\prime}-t\right)_{0},\left[\hat{H}^{\prime}, \hat{A}_{j}(\mathbf{r})\right]\right] \times\right. \\
& \left.\bar{\rho}(t, 0) \rho_{R}\right\} \text {, } \\
& { }_{I I} J_{j}^{(2)}(\mathbf{r}, t)=\frac{1}{i \hbar} \sum_{\mathbf{k}} \int_{-\infty}^{t} d t^{\prime} e^{\varepsilon\left(t^{\prime}-t\right)} \times \\
& \operatorname{Tr}\left\{\left[\hat{H}^{\prime}\left(t^{\prime}-t\right)_{0}, \hat{A}_{k}(\mathbf{r})\right] \bar{\rho}(t, 0) \times\right. \\
& \left.\rho_{R}\right\} \frac{\delta J_{j}^{(1)}(\mathbf{r}, t)}{\delta A_{k}(\mathbf{r}, t)},
\end{aligned}
$$

and we recall that $J_{j}^{(0)}$ and $J_{j}^{(1)}$, which in Mori's terminology [41] are called precession terms, are related to the nondissipative part of the motion, while dissipative effects are accounted for in $J_{j}^{(2)}$. Subindex nought indicates evolution in the interaction representation, $\delta$ indicates functional differentiation [66], it has been written for the Hamiltonian $\hat{H}=\hat{H}_{0}+\hat{H}^{\prime}$, where $\hat{H}_{0}$ stands for the kinetic energy and $\hat{H}^{\prime}$ contains the interaction potential energies.

This NESEF-based nonlinear kinetic theory allows for the derivation of generalized kinetic equations for far-fromequilibrium many body systems [71], which we briefly summarize. First it can bee noticed that dynamical processes in many-body systems that are described by kinetic equations are often dealt within certain approximations , e. g., Boltzmann equation for a dilute gas, Vlasov equation for a plasma, Landau equation for a weakly interacting gas, Focker-Planck equation for a Brownian particle, and so on [72]. Their common feature is that they involve the dynamics of singleparticle distributions. A question that has been one of the central themes of nonequilibrium statistical mechanics, ever since Boltzmann, is the one of giving solid foundations to kinetic equations from microscopic dynamics for, on the one hand, understanding their approximate validity and, on the other, to be able to go beyond removing the restrictions being imposed in the approximate treatments. Moreover, it has also been noticed that one of the complicated problems of the nonequilibrium theory of transport processes in dense gases and liquids is the fact that their kinetics and hydrodynamics are intimately coupled, and must be treated simultaneously (e.g., see Ref. [73]). On this we may say that microscopic descriptions of hydrodynamics, that is, associated to derivation of the kinetic equations from classical or quantum mechanics containing kinetic (transport) coefficients written in terms of correlation functions, is a traditional problem of long standing. An important aspect is the derivation of constitutive laws which express thermodynamic fluxes, as those of matter and energy, in terms of appropriate thermodynamic forces (typically gradients of densities as those of matter and energy). In their most general form these laws are nonlocal in space and non-instantaneous in time. The nonlocality is usually dealt with in terms of spatial Fourier transforms, and then the laws are expressed in the reciprocal space of wave-vectors $\mathbf{Q}$. A first kinetic-hydrodynamic approach can be considered to be the so-called classical (or Onsagerian) hydrodynamics; it gives foundations to, for example, the classical Fourier's and Fick's diffusion laws. But it works under quite restrictive conditions, namely, local equilibrium; linear relations between fluxes and thermodynamic forces (meaning weak amplitudes in the motion) with Onsager's symmetry laws holding; near homogeneous and static movement (meaning that the motion can be well described with basically Fourier components with long wavelengths and low frequencies, and then involves only smooth variation in space and time); weak and rapidly regressing fluctuations (e.g., Ref. [74]).

Hence, and under the pressure for obtaining a deep physical insight on the physical phenomena governing the processes that are fundamental to the development of modernday technologies with industrial/economic relevance, a satisfactory construction of kinetic theories is highly desirable. They should allow to deal with ultrafast (pico- and femtosecond scales) kinetic and relaxation processes, low dimensional and ultra-small (nanometric scale) systems, and displaying nonlinear behavior. Here are involved the technologies for electronic and opto-electronic devices, soft-matter engineering (polymers, micelles), food engineering, cosmetics, oil industry, and so on. It may be noticed that for systems in far-from-equilibrium conditions involving ultra-fast relaxation processes, and displaying nonlinear behavior, the emergence of instabilities and synergetic self-organization may eventually arise, as in cases in biophysics [75] and semiconductor physics, producing nonequilibrium Bose-Einsteinlike condensations [76]. We may also mention the case of laser-plasma interactions [77]. Furthermore, it can be noticed that the formalism presented below, can be extended to deal with the so-called non-conventional or "anomalous" situations which are associated to disordered media, consisting in systems showing a complex structure of a fractal-like (selfaffine in average) characteristics, whose range of applicability and of physical interest is large [23]. Fall on this area the distinctive behavior of polyatomic structures such as colloidal particles, surfactant micelles, and polymer and biopolymer (as DNA) molecules in a liquid, which are classical examples of what is presently referred to as soft condensed matter [24].

For illustration we consider the Hamiltonian dynamics of a system consisting of a fluid of interacting particles embedded in a fluid acting as a thermal bath, the latter at rest and in thermal equilibrium with an external reservoir [71]. The former is subjected to external forces - driving it out of equilibrium and the latter (the thermal bath) is taken in a state of constant equilibrium with an external thermal reservoir at temperature $T_{o}$. An analogous case, but at the quantum mechanical level, is the one of carriers embedded in the ionic lattice in doped or photoinjected semiconductors (see, for example Ref. [78]).

We write for the Hamiltonian

$$
H=H_{S}+H_{B}+W+H_{e x t},
$$

where,

$$
H_{S}=\sum_{j=1}^{N} \frac{p_{j}^{2}}{2 m}+\frac{1}{2} \sum_{j \neq k}^{N} V\left(\left|\mathbf{r}_{j}-\mathbf{r}_{k}\right|\right)
$$

is the one of the system of particles of mass $m$ consisting of the kinetic energy and their pair interaction via a central force 
potential;

$$
H_{B}=\sum_{\mu=1}^{N_{B}} \frac{P_{\mu}^{2}}{2 M}+\frac{1}{2} \sum_{\mu \neq v=1}^{N_{B}} \Phi_{B}\left(\left|\mathbf{R}_{\mu}-\mathbf{R}_{v}\right|\right)
$$

is the Hamiltonian of the bath particles of mass $M$, consisting of their kinetic energy plus their pair interaction via a central force potential ;

$$
W=\sum_{j, \mu} \Phi\left(\left|\mathbf{r}_{j}-\mathbf{R}_{\mu}\right|\right)
$$

is the interaction Hamiltonian of the particles with the thermal bath, and

$$
H_{\text {ext }}=\sum_{i} V_{\text {ext }}\left(\mathbf{r}_{i}, \mathbf{p}_{i}, t\right)
$$

is the Hamiltonian associated to the external force acting on the particles of the system, depending in its most general form on $\mathbf{r}, \mathbf{p}$ and $t$.

Under the stated condition that the bath (solvent) is in constant thermal equilibrium with an external reservoir at temperature $T_{o}$, its macroscopic state is characterized by the canonical distribution

$$
\begin{aligned}
\rho_{B}= & Z^{-1} \exp \left\{-\beta_{o}\left[\sum_{\mu=1}^{N_{B}} \frac{P_{\mu}^{2}}{2 M}+\right.\right. \\
& \left.\left.+\frac{1}{2} \sum_{\mu \neq v=1}^{N_{B}} \Phi_{B}\left(\left|\mathbf{R}_{\mu}-\mathbf{R}_{v}\right|\right)\right]\right\},
\end{aligned}
$$

where $\beta_{o}=\left[k_{B} T_{o}\right]^{-1}$ and $Z$ is the corresponding partition function. The auxiliary nonequilibrium statistical operator of the whole system has then the factorized form

$$
\bar{\Re}(t, 0)=\bar{\rho}(t, 0) \times \rho_{B},
$$

and for the auxiliary statistical operator of the system, $\bar{\rho}$, we take the one of Eq. (16), i.e. in terms of $\widehat{n}_{1}$ and $\widehat{n}_{2}$ of Eqs. (1) and (2) or (3) and (4). But, in a first approach it is supposed that the nonequilibrium state of the system is specified by the single-particle distribution function $f_{1}(\mathbf{r}, \mathbf{p} ; t)$ then, in that case, we choose the single particle phase density, $\widehat{n}_{1}(\mathbf{r}, \mathbf{p} \mid \Gamma)$, as the only relevant dynamical variable required. It means that we are considering dilute solutions (large distance in average between the system particles). Hence, the auxiliary nonequilibrium statistical operator for the particles embedded in the bath is

$$
\begin{aligned}
& \bar{\rho}(t, 0)= \exp \left\{-\phi(t)-\int d^{3} r \int d^{3} p \times\right. \\
&\left.F_{1}(\mathbf{r}, \mathbf{p} ; t) \widehat{n}_{1}(\mathbf{r}, \mathbf{p})\right\}=\prod_{j=1}^{N} \bar{\rho}_{j}(t, 0) \\
& \bar{\rho}_{j}(t, 0)=\exp \left\{-\phi_{j}(t)-\int d^{3} r \int d^{3} p F_{1}(\mathbf{r}, \mathbf{p} ; t) \times\right. \\
&\left.\delta\left(\mathbf{r}-\mathbf{r}_{j}\right) \delta\left(\mathbf{p}-\mathbf{p}_{j}\right)\right\},
\end{aligned}
$$

where $\bar{\rho}_{j}$ is a probability distribution for an individual particle, with $\phi(t)$ and $\phi_{j}(t)$ ensuring the normalization condition.
The average of $\widehat{n}_{1}$ over this NESEF-nonequilibrium ensemble, that is

$$
f_{1}(\mathbf{r}, \mathbf{p} ; t)=\operatorname{Tr}\left\{\widehat{n}_{1}(\mathbf{r}, \mathbf{p}) \rho_{\varepsilon}(t)\right\}=\operatorname{Tr}\left\{\widehat{n}_{1}(\mathbf{r}, \mathbf{p}) \bar{\rho}(t, 0)\right\}
$$

is a NESEF-based Boltzmann-type single-particle distribution function. The trace operation $T r$ in Eq. (39) is in this classical approach to be understood as an integration over phase space, and we recall that for the basic dynamical variables, and only for them, the trace with $\rho_{\varepsilon}(t)$ coincides with the one taken with $\bar{\rho}$.

According to Eq. (27), after identifying $A$ with $f_{1}(\mathbf{r}, \mathbf{p} ; t)$, we do have in the Markovian approximantion that $f_{1}$ satisfies the evolution equation

$$
\frac{\partial}{\partial t} f_{1}(\mathbf{r}, \mathbf{p} ; t)=J_{1}^{(0)}(\mathbf{r}, \mathbf{p} ; t)+J_{1}^{(1)}(\mathbf{r}, \mathbf{p} ; t)+J_{1}^{(2)}(\mathbf{r}, \mathbf{p} ; t) .
$$

The first two terms on the right are

$$
\begin{aligned}
J_{1}^{(0)}(\mathbf{r}, \mathbf{p} ; t) & =\operatorname{Tr}\left\{\left\{\widehat{n}_{1}(\mathbf{r}, \mathbf{p}), H_{o}\right\} \bar{\rho}(t, 0) \times \rho_{B}\right\}= \\
& =-(\mathbf{p} / m) \cdot \nabla f_{1}(\mathbf{r}, \mathbf{p} ; t),
\end{aligned}
$$

$$
\begin{aligned}
J_{1}^{(1)}(\mathbf{r}, \mathbf{p} ; t)= & \operatorname{Tr}\left\{\left\{\widehat{n}_{1}(\mathbf{r}, \mathbf{p}), H^{\prime}\right\} \bar{\rho}(t, 0) \times \rho_{B}\right\}= \\
= & -\nabla_{\mathbf{p}} V_{\text {ext }}(\mathbf{r}, \mathbf{p} ; t) \cdot \nabla f_{1}(\mathbf{r}, \mathbf{p} ; t)+ \\
& +\nabla V_{\text {ext }}(\mathbf{r}, \mathbf{p} ; t) \cdot \nabla_{\mathbf{p}} f_{1}(\mathbf{r}, \mathbf{p} ; t)+ \\
& +\nabla U(\mathbf{r} ; t) \cdot \nabla_{\mathbf{p}} f_{1}(\mathbf{r}, \mathbf{p} ; t),
\end{aligned}
$$

with

$$
U(\mathbf{r} ; t)=\int d^{3} r, \int d^{3} p^{\prime} V\left(\left|\mathbf{r}-\mathbf{r}^{\prime}\right|\right) f_{1}\left(\mathbf{r}^{\prime}, \mathbf{p}^{\prime} ; t\right)
$$

playing the role of a mean-field potential of interaction between the particles. The last term is the collision integral

$$
\begin{aligned}
J_{1}^{(2)}(\mathbf{r}, \mathbf{p} ; t)= & \int_{-\infty}^{t} d t^{\prime} e^{\varepsilon\left(t^{\prime}-t\right)} \operatorname{Tr}\left\{\left\{H^{\prime}\left(t^{\prime}-t\right)_{o},\right.\right. \\
& \left\{H^{\prime}, \widehat{n}_{1}(\mathbf{r}, \mathbf{p})\right\} \bar{\rho}(t, 0) \times \rho_{B}+ \\
& +\int_{-\infty}^{t} d t^{\prime} e^{\varepsilon\left(t^{\prime}-t\right)} \int d^{3} \mathbf{r}^{\prime} \int d^{3} p^{\prime} \times \\
& \left.\operatorname{Tr}\left\{\left\{H^{\prime}\left(t^{\prime}-t\right)_{o}, \widehat{n}_{1}\left(\mathbf{r}^{\prime}, \mathbf{p}^{\prime}\right)\right\} \bar{\rho}(t, 0)\right\}\right\} \times \\
& \left.\times \rho_{B}\right\} \frac{\delta J_{1}^{(1)}(\mathbf{r}, \mathbf{p} ; t)}{\delta f_{1}\left(\mathbf{r}^{\prime}, \mathbf{p}^{\prime} ; t\right)}
\end{aligned}
$$

where $H^{\prime}$ contains the potential energies $V$ and $\Phi$ of Eqs. (33) and (35), that is, the contributions to $J_{1}^{(2)}$ that come from the second order pair interactions between the system particles, and of the system particles with those of the thermal bath. Lengthy but straightforward calculations provide the following kinetic equation,

$$
\begin{aligned}
& \frac{\partial}{\partial t} f_{1}(\mathbf{r}, \mathbf{p} ; t)+\frac{\mathbf{P}(\mathbf{r}, \mathbf{p} ; t)}{m} \cdot \nabla f_{1}(\mathbf{r}, \mathbf{p} ; t)+ \\
& +\mathbf{F}(\mathbf{r}, \mathbf{p} ; t) \cdot \nabla_{\mathbf{p}} f_{1}(\mathbf{r}, \mathbf{p} ; t)- \\
& A_{2}^{[2]}(\mathbf{p}) \otimes\left[\nabla_{\mathbf{p}} \nabla\right] f_{1}(\mathbf{r}, \mathbf{p} ; t)- \\
& -B_{2}^{[2]}(\mathbf{p}) \otimes\left[\nabla_{\mathbf{p}} \nabla_{\mathbf{p}}\right] f_{1}(\mathbf{r}, \mathbf{p} ; t)-B(\mathbf{p}) f_{1}(\mathbf{r}, \mathbf{p} ; t)=
\end{aligned}
$$




$$
=J_{S}^{(2)}(\mathbf{r}, \mathbf{p} ; t),
$$

where

$$
\frac{\mathbf{P}(\mathbf{r}, \mathbf{p} ; t)}{m}=\frac{\mathbf{p}}{m}-\mathbf{A}_{1}(\mathbf{p})+\nabla_{\mathbf{p}} V_{e x t}(\mathbf{r}, \mathbf{p} ; t)
$$

$$
\mathbf{F}(\mathbf{r}, \mathbf{p} ; t)=-\nabla V_{e x t}(\mathbf{r}, \mathbf{p} ; t)-\mathbf{B}_{1}(\mathbf{p})-\mathbf{F}_{n l}(\mathbf{r} ; t)-\nabla U(\mathbf{r} ; t),
$$

$$
\begin{aligned}
\mathbf{F}_{n l}(\mathbf{r} ; t)= & \int d^{3} r^{\prime} \int d^{3} p^{\prime} f_{1}\left(\mathbf{r}^{\prime}, \mathbf{p}^{\prime} ; t\right) \times \\
& \mathbf{G}_{n l}\left(\mathbf{r}^{\prime}-\mathbf{r}, \mathbf{p}^{\prime}\right),
\end{aligned}
$$

In Eqs. (45) to (48) dot stands as usual for scalar product of vectors; we wrote $\otimes$ for fully contracted product of tensors, and $U(\mathbf{r} ; t)$ is given in Eq. (43). In these equations $\mathbf{A}_{1}, \mathbf{B}_{1}$, $\mathbf{G}_{n l}, A_{2}^{[2]}, B_{2}^{[2]}$ and $B$ are kinetic coefficients whose detailed expressions we omit to write down (they contain the square modulus of the Fourier transform of the particle-bath interaction), and $J_{S}$ is the collision integral arising out of the two particle interaction which takes the form in this case of one in the weak-coupling limit (complete details in Ref. [71]).

When Eq. (45) is compared with the, say, standard Boltzmann kinetic equation, it contains several additional interesting contributions. First, $\mathbf{P}(\mathbf{r}, \mathbf{p} ; t)$, Eq. (46), can be interpreted as a modified momentum, composed of the linear one, $\mathbf{p}$, plus a contribution arising out of the interaction with the thermal bath, $m \mathbf{A}_{1}(\mathbf{p})$, and another given by the $\mathbf{p}$-gradient of the potential energy associated to the external force (if it depends on $\mathbf{p})$. The force $\mathbf{F}(\mathbf{r}, \mathbf{p} ; t)$, see Eq. (47), is composed of the external force, plus $\mathbf{B}_{1}(\mathbf{p})$ arising out of the interaction with the thermal bath. Also we have an interesting third one, $\mathbf{F}_{n l}(\mathbf{r} ; t)$, which is an effective force between pairs of particles generated through the interaction of each of the pair with the thermal bath. A similar presence of an induced effective coupling of this type has been evidenced in the case of two Brownian particles embedded in a thermal bath [79] and also, it is of the same kind that leads to the formation of Cooper pairs in type-I superconductivity, in the hydrodynamic of polymer solutions, etc. Such contribution is of a nonlinear (bilinear in $f_{1}$ ) character, and the last is the Vlasov force term. The fourth and fifth terms on the left of Eq. (45) consists of a cross double differentiation, $\left[\nabla_{\mathbf{p}} \nabla\right]$, which take account of cross effects of anisotropy caused by non-uniformity, and a double differentiation in the momentum variable, $\left[\nabla_{\mathbf{p}} \nabla_{\mathbf{p}}\right]$, of $f_{1}$ related to anisotropy and diffusion in momentum space; both are consequence of the presence of the interaction with the thermal bath. The last term on the left side, proportional to the distribution, is a kind of source term with a rate of pumping $B^{-1}$, which together with the force term $\mathbf{B}_{1}(\mathbf{p})$ give rise to the dynamic frictional force in the Brownian limit. Finally, on the right is the contribution associated to the system pair particle collisions in the weak coupling limit. This is equivalent to the so-called weakly coupled gas collision integral (e.g. [80]). Hence, to go beyond the Markovian approximation, one needs to go back to the kinetic equation (45) [71] to include memory and vertex renormalization effects and higher order collision integrals.

Moreover, Eq. (45) embraces two particular results. The first is the Markovian approximation of a weakly coupled closed gas which we get turning off the interaction with thermal bath, i.e., setting $\Phi=0$. Balescu [80] has obtained the same equation using the dynamics of correlations approach of the Brussels school. Zubarev et al. [45] obtained the same equation exploring the classical diagram technique to handle the hierarchy of equations for the correlation functions. The second is a generalization of the kinetic equation known as the Kramers-Chendrasekhar-Fokker-Planck [81]. We get this result setting to zero the interaction $V\left(\left|\mathbf{r}_{j}-\mathbf{r}_{k}\right|\right)$ of Eq. (33). Here we have derived the same equation using the NESEF method which, we believe, contributes to clarify the equivalence of these several approaches.

A last remark: knowledge of $f_{1}(\mathbf{r}, \mathbf{p} ; t)$ implies complete information about the actual distribution of particles, and therefore of the physical properties of the system. Alternatively, knowing all the moments of the distribution allows to have a complete knowledge of its characteristics. A knowledge of some moments is not sufficient to determine the distribution completely; it implies in only possessing partial knowledge of the characteristics of this distribution [81]. On this H. Grad noticed that the question of the general solutions of the standard Boltzmann equation can be tackled along two distinct lines. One is to attempt to solve Boltzmann equation for the distribution $f_{1}$ itself in specific problems. Other is to obtain new phenomenological equations which generalize the usual (classical-Onsagerian) fluid dynamical equations. The aim is to show the transition from the Boltzmann equation in which a state is given by $f_{1}(\mathbf{r}, \mathbf{p} ; t)$ to the conventional fluid description in which a state is given by the density $n(\mathbf{r}, t)$, the velocity field $\mathbf{v}(\mathbf{r}, t)$, and the stress tensor $T^{[2]}(\mathbf{r}, t)$, in a sufficient generality to cover a broad class of problems. This approach was initiated by Maxwell [82] and continued by Grad [83] (it was called Grad's moments procedure [84]). In this vein, Eq. (45) can be associated with a quite extensive generalization of this moments procedure, consisting into introducing the full set of moments of $f_{1}(\mathbf{r}, \mathbf{p} ; t)$ in the variable p. These moments produce quantities with a clear physical meaning, namely, the densities of particles and of energy and their fluxes of all order, namely, the two vectorial fluxes, or currents, the tensorial fluxes, beginning with the second-order tensor flux (which is related to the pressure tensor), and all the other higher-order fluxes. In that way we obtain a quite generalized Nonlinear Higher-Order Hydrodynamics, coupled to a Non-Equilibrium Thermodynamics, all together in the kinetic approach provided by NESEF, and where it can be evidenced the presence and influence of the several contributions above described [85].

\section{ON PROCESSES INVOLVED IN PRESENT DAY ADVANCED TECHNOLOGIES AND COMPETITIVE INDUSTRIES}

Such processes are associated to, in general, optical and transport properties in systems away from equilibrium, displaying ultrafast relaxation processes, being in constrained geometries, and presenting nonlinear behavior. This is to be expected in multiple situations involving technological applications and manufacturing processes. Also, when applying the theoretical formalism to these questions one is making contact with the fundamental point in the scientific method 
of corroborating theory through comparison with experiment [29]. It is worth mentioning S. J. Gould's observation that "a detail, by itself, is blind; a concept without a concrete illustration is empty [...] Darwin, who had such keen understanding of fruitful procedure in science, knew in his guts that theory and observation are Siamese twins, inextricably intertwined and continually interacting" (emphasis is ours) [86]. In particular, in the present question of statistical thermodynamics we restate the call of Ryogo Kubo, who expressed that "statistical mechanics has been considered a theoretical endeavor. However, statistical mechanics exists for the sake of the real world, not for fictions. Further progress can only be hoped by close cooperation with experiment" [30].

As pointed out by several authors in multiple occasions, any theory in Physics, besides being logically consistent and mathematically correct, requires fundamentally for its validation the comparison with experimental data, and of course, to show a good agreement between calculated and measured values of observables, as well to properly predict new results and phenomena (e.g. [87-90]).

It is worth citing Stephen Hawking [91] who expressed that "I do not demand that a theory corresponds to reality. But that does not bother me. I do not demand that a theory corresponds to reality because I do not know what it is. Reality is not a quality you can test with litmus paper. All I am concerned with is that the theory should predict the results of measurement".

Imagination and boldness in making theory must always be policed by experience. As the German painter Ferdinand Lèger (1881-1955) put it, "To be free and yet not lose touch with reality, that is the drama of that epic figure who is variously called scientist, artist, or poet" (quoted and translated by Douglas Cooper in Ferdinand Lèger and the nouvelle space).

The pioneers Bacon, Galileo, and Newton were of this persuasion; Harvey (in his treatise on the circulation of blood) elegantly and concisely summarized the point in his statement that "the facts cognizable by the senses wait upon no opinions, and the works of nature bow to no antiquity..." (quoted in Ref. [88]).

We clarify that what is done in the following Sections is, in a sense, to present a kind of catalog of a certain chosen set of experiments, which have been theoretically dealt with in the framework provided by NESEF. No detail of the experiments and the calculations is presented, what is unnecessary once this is done in published articles, and then, for details, we refer the reader to them, which are in each case indicated in the text and listed in the bibliography.

Seven areas of particular interest where the formalism has full and quite useful application are those indicated below on which a vast amount of very successful experimental studies is available in the scientific literature on the subjects. We shall concentrate the attention on a description of some examples for the sake of illustration of the workings of the formalism. A number of other applications, including a report on earlier applications of the formalism, is described in the review article of Ref. [92].

In the following Sections we very briefly describe several examples of optical and transport properties in systems away from equilibrium showing ultrafast relaxation processes, constrained geometries, and nonlinear behavior, to be expected in working conditions of devices in technological applications and their end use. They are:

\section{Ultrafast Relaxation Processes in Semiconductors}

VII. Low-Dimensional Semiconductor Devices

VIII. Nonlinear Transport in Highly-Polar Semiconductors

IX. Nonlinear Higher-Order Thermo-Hydrodynamics

\section{Coherent States and Nonequilibrium Bose-Einstein-like} Condensations

\section{Thermo-Statistics of Complex Structured Systems}

XII. Nonconventional Thermo-Hydrodynamics

\section{ULTRAFAST RELAXATION PROCESSES IN SEMICONDUCTORS}

In this Section we present some general comments on the thermo-statistical aspects of the ultrafast evolution of the nonequilibrium state of highly photoexcited polar semiconductors. These processes can be evidenced, and its evolution followed, in experiments of ultrafast laser spectroscopy, which is an excellent technique for also studying other systems, e.g., biological systems, polymers, etc. [93-99]. On this we reproduce parts of the Introduction of the article "Big Payoffs in a Flash" by J.M. Hopkins and W. Sibbett in Scientific American, September 2000 issue [97], who wrote that

"How long did it take you to read this sentence? Just recognizing the first letter took only milliseconds. Around 0.05 millisecond, or 50 microseconds, passes each time chemicals diffuse across a synapse, carrying a signal from one neuron to another in your brain. Are you holding the magazine at a comfortable reading distance? It takes light one or two nanoseconds to travel from the page to your eye and about 20 picoseconds to pass through the lens in your eye. And yet these brief natural events are epically long compared with the shortest man-made events, which proceed 1,000-fold more swiftly: pulses of laser light that last for only a few femtoseconds (quadrillionths of a second). The science and technology of ultrashort-pulse lasers have enjoyed much exciting progress since they were developed in the mid-1960s. In particular, the past decade has seen pulses shorter than 10 femtoseconds and the emergence of a new generation of versatile, compact ultrashort-pulse lasers a revolutionary change from their large, temperamental, power-hungry ancestors. Such laser designs, which make use of sophisticated nonlinear optical phenomena and concurrent advances in diode lasers, increasingly meet the stringent specifications and reliability necessary for many industrial and medical applications. As we enter the 21 st century, ultrashort-pulse lasers are becoming more impressive in scope and intensity, producing beams that span the electromagnetic spectrum from X-rays to $T$-rays (terahertz radiation, beyond infrared) and generating optical peak powers as colossal as petawatts (billions of megawatts). As a result, many new applications in physics, chemistry, biology, medicine, and digital optical technology are emerging and attracting worldwide interest in science and industry."

Studies of the optical and transport properties of semiconductors under high levels of excitation have shown a pleiad of novel and quite interesting features evidenced in ultrafast 
laser spectroscopy (UFLS). This powerful experimental technique involves the interaction of matter with radiation, which is one of the most studied areas of physics and has played a decisive role in the development of modern physics at the beginning of the XX century. However, until the second half of that century, all processes investigated have been associated with weak radiation fields for which the usual perturbation theory, and the accompanying linear response theory near equilibrium, is applicable. Although this approach has been remarkably successful in explaining a great variety of phenomena, in the last decades - and in the present one approaching the end of the twentieth century - the new and greatly improved technological situation involving the advent of lasers, providing us with sources of intense electromagnetic radiation, requires new and sophisticated theoretical approaches, that is, a response theory capable to deal with arbitrarily far-from-equilibrium systems. Moreover, the notable improvements in time-resolved laser spectroscopy have made it a very useful tool to be used with a high degree of confidence in the investigation of very rapid microscopic mechanisms in the physical and biological realms (see for example Refs. [93-99]).

In particular, ultrafast responses and functioning under farfrom-equilibrium conditions in semiconductor systems pose new, interesting, and quite engaging problems in the physics of condensed matter. These systems, as we have already emphasized in several occasions, become an extremely useful testing ground for theoretical ideas in the domain of nonequilibrium statistical thermodynamics of many-body systems. Besides the interest in the comprehension of the basic physical principles underlying these significant situations, there exists a parallel relevant technological interest arising out of the fact that semiconductors working in nonequilibrium conditions have multiple practical applications in electronic devices. Picosecond and femtosecond laser spectroscopy allows to probe ultrafast nonlinear irreversible processes in matter, thus providing an extremely adequate and sophisticated experimental instrument for the study of the nonequilibrium thermodynamic evolution of highly excited semiconductor samples $[78,99,100]$.

The theories appropriate for the treatment of these farfrom-equilibrium many-body systems ought to make it possible to determine the detailed time evolution of the nonlinear irreversible processes that take place in the system while it is probed. This is a quite attractive and actual problem connected with the nonequilibrium nonlinear statistical mechanics and thermodynamics of dynamical processes. UFLS studies of the highly photoexcited plasma in semiconductors (HEPS, which consists of electron and hole pairs - as mobile carriers - created by an intense laser pulse which are moving in the background of lattice vibrations) have received particular attention along the last decades. These studies provide information on the kinetic of relaxation of the photoexcited carriers and of the nonequilibrium phonon field, as well as on ultrafast transient transport. A detailed description is given in the special issue on UFLS of the Braz. J. Phys. (vol. 26, no. 2) [100].

\section{LOW-DIMENSIONAL SEMICONDUCTOR DEVICES}

Terrel Hill noticed in 1962 [101] that there are some important respects in which small thermodynamic systems differ experimentally from macroscopic systems. In general, measurements are not made on a single small system but on a large number of small systems. An example is a very dilute solution of a macromolecule: the "small system" is one macromolecule; the solution contains many macromolecules; but the solution must be very dilute as that the macromolecules do not interact with each other. Moreover, a macroscopic system immersed in a reservoir may exchange heat, molecules, etc., with the reservoir, but the intermolecular interaction between the system and the reservoir at the surface of contact is of negligible order. This is, in general, no longer the case for a small system immersed in a solvent. Also, certain properties which can be varied experimentally at will for a macroscopic system cannot be so varied with a small system, e.g., the volume of a colloidal particle.

Nowadays this question has acquired an enormous relevance because of the technological and commercial interest it involves, which extends to the areas of biology and medicine.

Mihail Roco, in an article in Forum Section of Scientific American [102], notice that:

"Today nanotechnology is still in a formative phase - not unlike the condition of computer science in the 1960s or biotechnology in the 1980s. Yet it is maturing rapidly. Between 1997 and 2005, investment in nanotech research and development by governments around the world soared from $\$ 432$ million to about $\$ 4.1$ billion, and corresponding industry investment exceeded that of governments by 2005. By 2015, products incorporating nanotech will contribute approximately \$1 trillion to the global economy. About two million workers will be employed in nanotech industries, and three times that many will have supporting jobs [...] Over the next couple of decades, nanotech will evolve through four overlapping stages of industrial prototyping and early commercialization. The first one, which began after 2000, involves the development of passive nanostructures: materials with steady structutures and functions, often used as parts of a product. The second stage, which began in 2005, focuses on active nanostructures that change size, shape, conductivity or other properties during use. New drug-delivery particles could release therapeutic molecules in the body only after they reached their targeted diseased tissues. Electronic components such as transistors and amplifiers with adaptive functions could be reduced to single, complex molecules. Starting 2010, workers will cultivate expertise with systems of nanostructures, directing large numbers of intricate components to specified ends. One application could involve the guided self-assembly of nanoelectronic components into three-dimensional circuits and whole devices. Medicine could employ such systems to improve the tissue compatibility of implants, or to create scaffolds for tissue regeneration, or perhaps even to build artificial organs. After 2015-2020, the field will expand to include molecular nanosystems - heterogeneous networks in which molecules and supramolecular structures serve as distinct devices. The proteins inside cells work together this way, but whereas biological systems are water-based and markedly temperature-sensitive, these molecular nanosystems will be able to operate in a far wider range of environments and should be much faster. Computers and robots could be reduced to extraordinarily small sizes. Medical applications might be as ambitious as new types of genetic therapies and antiaging treatments. New interfaces linking people directly to 
electronics could change telecommunications. Over time, therefore, nanotechnology should benefit every industrial sector and health care field. It should also help the environment through more efficient use of resources and better methods of pollution control."

In this field one has to deal with systems in which one or more directions are in the nanometric space scale (e.g., quantum wells, 2D, quantum wires, 1D, quantum dots, 0D), which as a rule exhibit, significantly, the novel phenomena and processes. As noticed, the field is present in modern technologies.

The topic is further considered at the end of Section XI, once in small systems boundary conditions are of large relevance in determining the physical properties of the system and, therefore, its end-use utility. The interfaces always present a structure (rugousness) with characteristic in a nano and subnanometer scale, thus of the order of the space extension of the material [23]. Because the researcher does not have in general an access to the details of the surface structure it is said that we have in hands a problem with "hidden constraints". In Section XI two cases of technolog$\mathrm{ical} /$ industrial/commercial interest are presented, namely, the characterization of nanometric quantum wells in semiconductor heterostructures by optical spectroscopy, and characterization of nanometric electrodes in microbatteries.

\section{NONLINEAR TRANSPORT IN HIGHLY-POLAR SEMICONDUCTORS}

In a general article in Physics Today, issue of October 2000 [103], it was stated that: "The recent achievement of compact blue-emitting gallium nitride semiconductor lasers is likely to have far-reaching technological and commercial effects. The lasers' short wavelengths - around $400 \mathrm{~nm}$, half that of gallium arsenide-based lasers, permit higher spatial resolution in applications such as optical storage and printing. And the high photon energy will open up new applications for these inexpensive, compact light sources. An aesthetic satisfaction with these devices stems from finally extending the existing and mature semiconductor laser technology for the near-infrared and red to encompass the "new frontier" of blue and near-ultraviolet regions, thereby bridging the entire visible spectrum". It was also commented that: "At the same time, there are significant research opportunities arising from a plethora of poorly understood microscopic issues in the underlying material system, which include such fundamental properties as charge control, transport, and formation of optical gain for stimulated emission".

Moreover, it was there called the attention to the facts that one major commercial impact of blue diode lasers is to occur in high-density optical storage in digital versatile disks. Moreover, in the case of color projection displays and highresolution laser printers. Also a huge technological opportunity is offered by GaN-based light-emitting diodes (LEDs), in which incoherent light is produced by spontaneous as opposed to stimulated emission.

As example of commercial uses were indicated the case of green LEDs for traffic lights and violet LEDs that can be combined with phosphors to produce white lighting, a possible replacement for the ubiquitous incandescent lightbulb, and blue and green nitride LEDs for being integrated into large-scale outdoor displays.

It is also worth noticing a journalistic article in Scientific American (August 2000) [104], from where we extract the sentence that: "For more than 25 years, LEDs were like a third of a rainbow. Red, orange, yellow, and the yellowish green were all you could get. Engineers wanted blue and true green because with those colors, along with the red they already had, they could built fabulous things, such as whitelight-emitting devices as much as 12 times more efficient and longer-lasting than ordinary lightbulbs. Small wonder, then, that analysts say LEDs are poised to revolutionize the lighting industry and more beyond their familiar role as mere indicator light. In the mean time, colored LEDs are being deployed as traffic lights and in displays, the biggest being the eightstory-tall Nasdaq display in New York City's Time Square. And a blue semiconductor laser, similar to a LED, will soon quadruple the storage of capacity of DVD and CD players and the resolution of laser prints. Most of the milestones on the way to these optoelectronics triumphs took place, oddly enough, on the island of Shikoku, something of a backwater in the Japanese chain. [from Nichia Corporation, a once obscure Japanese maker of phosphors for cathode-ray tubes and fluorescent lights]."

The recent successful commercialization of III-Nitridesbased light-emitting diodes in the blue-yellow range, and the development of injection lasers and ultraviolet detectors, drove initially the focus of basic research on these wide-gap semiconductors to the understanding of their optical properties [105-108]. However, soon it was also recognized their favorable properties for the implementation of electronicpower devices, high-performance and high-frequency transistors [109-113], with superior characteristics than those based on silicon $[114,115]$. The efforts undertaken on the investigation of the III-Nitrides steady-state transport properties, looking for a better determination of bulk material parameters and band structure [116], are relevant for establishing their figure of merit for electronic devices with certain confidence. However, despite the improved understanding of the steadystate transport properties of III-Nitrides obtained in the last few years, the agreement of the carriers' mobility data with theoretical descriptions varies from broad to very good [117]; only a qualitative agreement was obtained between theoretical predictions and the recent measurements of the electron velocity-field characteristics in GaN [118].

Focus on the improvement of III-Nitrides-based devices with submicron channels has stimulated research on their transient-transport properties, since in such cases the carriers may not attain the steady-state transport regime. The possibility of transient ballistic transport in $\mathrm{GaN}$ was demonstrated to occur for applied electric fields greater than $140 \mathrm{kV} / \mathrm{cm}$ [119], and investigations on the transient transport regime have indicated the possibility of existence of an overshoot effect in both the electron-drift velocity [120-123] and mean energy [123]. In the work of Ref. [123], the explanation for the existence of the overshoot effect in the III-Nitrides, in conditions where intervalley scattering is negligible, was considered to be due to the interplay of energy and momentum relaxation times: no overshoot occurs when the momentum relaxation time, which is smaller than the energy relaxation time shortly after application of the electric field, becomes predominantly larger than the other; on the other hand, the overshoot fol- 
lows at intermediate to high fields when the relaxation time for energy remains larger than the one for momentum. But, no detailed explanation was presented confirming the validity of the above arguments.

In Ref. [124] we present such detailed derivation, obtaining analytical expressions for the so-called characteristic time for conduction (or current) and the momentum relaxation time, from which the conditions for the onset of the electrons' driftvelocity overshoot, in the case of these polar semiconductors in the parabolic-conduction band approximation can be clearly evidenced. For that purpose we obtain the equations of evolution for the energy of electrons and longitudinal-optical and acoustic phonons, $E_{c}(t), E_{L O}(t), E_{A C}(t)$ (the TO phonons are ignored, once they weakly interact with the electrons in the conduction band), and the electrons' momentum $\mathbf{P}(t)$. In summary, we may say that as a general rule, in the study of transport phenomena analytical-type methods have been based on Boltzmann-like transport theories, which, however, have limitations when nonlinear effects become to have relevance. Thus, improved analytical methods, that is nonlinear quantum kinetic theories for studying physical phenomena in systems arbitrarily away from equilibrium, are desirable. An advantage over Monte Carlo computational methods resides in that the analytical equations may provide a better physical insight and interpretation of the results. This is the NESEFbased nonlinear kinetic theory described in Sections III and IV.

The study of transport properties of semiconductors under high level of excitation, eventually following nonlinear laws, are of great interest not only for its relevance in the functioning of electronic and optoeletronic devices, but also because of providing an excellent testing ground for theoretical ideas in the field of many-body systems in far-from-equilibrium conditions as we do here. Hence, as noticed, nonlinearities are present in both transport properties and relaxation processes, which may give origin to new and interesting phenomena.

Let us consider the case of polar semiconductors described by a two-inverted-parabolic bands model (in the effective mass approximation, and conduction band secondary valleys are ignored), where a concentration $n_{e}$ of electrons or $n_{h}$ of holes has been created by doping. A constant electric field of intensity $F$ in, say, $x$-direction is applied, which accelerates these carriers ("hot" carriers) while there follows a transferring of their energy and momentum (in excess of equilibrium) to the phonon field. The sample is in contact with a thermal reservoir at temperature $T_{0}$, with the phonons being warmed up in scattering events involving Fröhlich, deformation potential, and piezoelectric interactions with the hot carriers. Scattering by impurities is neglected in comparison with the one due to lattice vibrations.

The Hamiltonians takes the form $\hat{H}_{0}+\hat{H}^{\prime}$, with $\hat{H}_{0}$ consisting of the free Hamiltonians of the carriers (electrons or holes) and of the phonons, while $\hat{H}^{\prime}$ contains the carrierphonon interactions and the coupling with the external electric field. Coulomb interaction between carriers (contributing to very fast - subpicosecond scale - relaxation processes) is incorporated in $\hat{H}_{0}$ but through the random phase approximation. For the basic microdynamical variables, as noticed above, are chosen the free Hamiltonian $\hat{H}_{0}$ (the one of the carriers $\hat{H}_{a}$ and those of the phonons $\left.\hat{H}_{L O}, \hat{H}_{T O}, \hat{H}_{A}\right)$ and the particle number $\hat{N}_{a}$ ( $a=e$ or $h$ for electrons and holes respectively). Next, on the basis that the electric field produces a current it is introduced the linear momentum $\hat{\mathbf{P}}_{a}$ (which is the flux of mass). Consequently, the basic macrodynamical variable are [78]

$$
\left\{E_{a}(t), \mathbf{P}_{a}(t), E_{L O}(t), E_{T O}(t), E_{A}\right\},
$$

whose evolution equations characterize the macroscopic evolution of the system. They are:

$$
\begin{gathered}
\frac{d}{d t} E_{a}(t)=\frac{e_{a} \mathbf{F}}{m_{a}^{*}} \cdot \mathbf{P}_{a}(t)-\left|J_{E_{a}}^{(2)}(t)\right|, \\
\frac{d}{d t} \mathbf{P}_{a}(t)=n_{a} V e_{a} \mathbf{F}+\left|\mathbf{J}_{\mathbf{P}_{a}}^{(2)}(t)\right|, \\
\frac{d}{d t} E_{L O}(t)=\left|J_{E_{L O}}^{(2)}(t)\right|+\left|J_{E_{L O, A}}^{(2)}(t)\right|, \\
\frac{d}{d t} E_{T O}(t)=\left|J_{E_{T O}}^{(2)}(t)\right|+\left|J_{E_{T O, A}}^{(2)}(t)\right|,
\end{gathered}
$$

where $V$ is the volume of sample and $e_{e}=-e$ and $e_{h}=e$ and we recall that the $A$ phonons remain in equilibrium at temperature $T_{0}$, and then $E_{A}$ is constant in time. In Eq. (49) the first term on the right accounts for the pumping of energy on the carrier system arising out of the presence of the electric field, while the second represents the rate of excess energy transferred to the lattice (LO, TO and A phonons).

In Eq. (50) the first term on the right is the force produced by the presence of the electric field, and the second the rate of momentum transferred to the lattice. In Eqs. (51) and (52) we have the term of gain of energy pumped on the phonons by the nonequilibrated ("hot") carriers and the transfer of such energy to the A phonons (at temperature $T_{0}$ ), the latter acting as a thermal bath.

Defining time-dependent carrier-momentum $\tau_{P_{a}}(t)$ and carrier-energy $\tau_{E_{a}}(t)$ relaxation times as

$$
\begin{gathered}
\left|\mathbf{J}_{\mathbf{P}_{a}}^{(2)}(t)\right| \equiv n_{a} V m_{a}^{*} \frac{\left|v_{a}(t)\right|}{\tau_{P_{a}}(t)}, \\
\left|J_{E_{a}}^{(2)}(t)\right|=\frac{E_{a}(t)-E_{a}^{e q}}{\tau_{E_{a}}(t)},
\end{gathered}
$$

where $v_{a}(t)$ is the drift velocity in the direction of the electric field (i.e. $P_{\alpha}(t)=m_{\alpha} v_{\alpha}(t)$ ) and $E_{a}^{e q}$ the energy of the carriers in equilibrium with the A phonons (and then the reservoir) at temperature $T_{0}$.

Using Eqs. (53) and (50) we find an equation of evolution for the drift velocity, namely

$$
\frac{d}{d t} \mathbf{v}_{a}(t)=\left(e_{a} / m_{a}^{*}\right) \mathbf{F}-\frac{\mathbf{v}_{a}(t)}{\tau_{P_{a}}(t)},
$$

which is an equation of the Newton-Langevin type but with a time-dependent relaxation time (evolving in time with the macroscopic state of the system). Equation (55) can be alternatively written in the form of the integral equation

$$
\mathbf{v}_{a}(t)=\left(e_{a} / m_{a}^{*}\right) \mathbf{F} \tau_{c a}(t),
$$


where

$$
\tau_{c a}(t)=\exp \left\{-\Psi_{a}(t)\right\} \int_{0}^{t} \exp \left\{\Psi_{a}\left(t^{\prime}\right)\right\} d t^{\prime},
$$

with

$$
\Psi_{a}(t)=\int_{0}^{t} d t^{\prime}\left(\tau_{P_{a}}\left(t^{\prime}\right)\right)^{-1},
$$

and it has been taken the initial condition $v_{a}(0)=0$. Moreover, defining the current density

$$
\mathbf{I}_{a}(t)=n_{a} e_{a} \mathbf{v}_{a}(t),
$$

after replacing in it the expression of Eq. (56) we have that

$$
\mathbf{I}_{a}(t)=\sigma_{a}(t) \mathbf{F},
$$

thus introducing a time-dependent Drude-type conductivity, namely

$$
\sigma_{a}(t)=\left(n_{a} e^{2} / m_{a}^{*}\right) \tau_{c a}(t),
$$

and we call $\tau_{c a}(t)$ the characteristic time for conduction, which is dependent on the evolution of the macroscopic (nonequilibrium thermodynamic) state of the system.

This NESEF-based nonlinear transport theory has been successfully applied to the study of carriers in polar semiconductors (e.g., GaAs, GaN, and others) under intermediate to strong electric fields $[123,125]$.

\section{NONLINEAR HIGHER-ORDER THERMO-HYDRODYNAMICS AND NON-NEWTONIAN RHEOLOGY}

Hydrodynamics constitutes an old field of scientific and technical interest, and within its domain we can notice the work of the great polymath and artistic genius Leonardo da Vinci (1452-1519) in the fifteen century. It has been written [126] that "Throughout his life, Leonardo was obsessed with the movement of water, and there were many occasions when he turned this interest to useful ends".

Nowadays, Hydrodynamics also enters into the line of a discipline in search for a large improvement in order to cover the theme of this publication, namely, to accompany the notable developments in technology, and the pursue of continuous improvements in industrial processes, looking for more efficient productivity and better quality. Evidently, several areas are involved, and we can mention, hydraulic engineering, ecology, food engineering, soft-matter (including polymers) engineering, petroleum production, aerospatial engineering, and so on and so forth.

We begin noticing that, as a rule, what is involved consists in dealing with the transport of fluids, and on that it has been noticed that one of the complicated problems of the nonequilibrium theory of transport processes in dense gases and liquids is the fact that their kinetics and hydrodynamics are intimately coupled, and must be treated simultaneously (e.g., see Ref. [45,73,74,127-129]). On this we may say that microscopic descriptions of hydrodynamics, that is, associated to derivation of kinetic equations from classical or quantum mechanics containing kinetic (transport) coefficients written in terms of correlation functions, is a traditional problem of long standing. An important aspect is the derivation of constitutive laws which express thermodynamic fluxes, as those of matter and energy, in terms of appropriate thermodynamic forces (typically gradients of densities as those of matter and energy). In their most general form these laws are nonlocal in space and non-instantaneous in time. The nonlocality is usually dealt with in terms of spatial Fourier transforms, and then the laws are expressed in the reciprocal space of wave-vectors $\mathbf{Q}$. A first kinetic-hydrodynamic approach can be considered to be the so-called classical (or Onsagerian) hydrodynamics; it gives foundations to, for example, the classical Fourier's and Fick's diffusion laws. But it works under quite restrictive conditions, namely, local equilibrium; linear relations between fluxes and thermodynamic forces (meaning weak amplitudes in the motion) with Onsager's symmetry laws holding; near homogeneous and static movement (meaning that the motion can be well described with basically Fourier components with long wavelengths and low frequencies, and then involves only smooth variation in space and time); weak and rapidly regressing fluctuations (see, for example, Refs. [74,130]).

Hence, more advanced approaches are required to lift these restrictions. Consider first near homogeneity, which implies validity in the limit of long wavelengths (or wavenumbers $Q$ approaching zero), and to go beyond it is necessary to introduce a proper dependence on $Q$ valid, in principle, for intermediate and short wavelengths (intermediate to large wavenumbers). In phenomenological theories this corresponds to go from classical irreversible thermodynamics to extended irreversible thermodynamics [12,131,132]. This is what has been called generalized hydrodynamics, a question extensively debated for decades by the Statistical Mechanics community. Several approaches have been used, and a description can be consulted in Chapter 6 of the classical book on the subject by Boon and Yip [127]. Introduction of nonlocal effects for describing motions with influence of ever decreasing wavelengths, going towards the very short limit, has been done in terms of expansions in increasing powers of the wavenumber, which consists in what is nowadays sometimes referred to as higher-order hydrodynamics $(\mathrm{HOH})$. Attempts to perform such expansions are the so-called Burnett and super-Burnett approaches in the case of mass motion, and Guyer-Krumhansl approach in the case of propagation of energy (see for example Refs. [133,134]). An usual approach has been based on the moments solution procedure of Boltzmann equation, as in the work of Hess [135], using a higher-order Chapman-Enskog solution method. The Chapman-Enskog method provides a solution to Boltzmann equation consisting of a series in powers of the Knudsen number, $K_{n}$, given by the ratio between the mean-free path of the particles and the scale of change (relevant wavelengths in the motion) of the hydrodynamic fields. Retaining the term linear in $K_{n}$ there follows Navier-Stokes equation, the term in $K_{n}^{2}$ introduces Burnett-like contributions, and the higher-order ones ( $K_{n}^{3}$ and up) the super-Burnett contributions.

A satisfactory development of a $\mathrm{HOH}$ being also nonlinear and including fluctuations is highly desirable for covering a large class of hydrodynamic situations, and, besides 
its own scientific interest, also for obtaining insights into technological-industrial processes having an associated economic interest. Also, we can mention its fundamental relevance in Oceanography and Metereology (e.g. [136,137]), and that it has been stated [138] that the idea of promoting hydraulics by statistical inference is appealing because the complete information about phenomena in hydraulics seldom exists, for example sediment transport, and also the more fundamental problem in fluid mechanics of describing the velocity distribution in fluids under flow [139-141]. Indeed, the nonlocal terms become specially important in miniaturized devices at submicronic lengths (e.g., [142]), or in the design of stratospheric planes, which fly in rarefied gases in a density regime between the independent particle description and the purely continuous description. Another particular problem to it related is the one of obtaining the structures of shock waves in fluids for wide ranges of Mach numbers (e.g., [143]). Moreover, Burnett approximation of hydrodynamics has been shown to provide substantial improvement on many features of the flow occurring in several problems in hydrodynamics, e.g. the case of Poiseuille flow [144] and others [145].

The microscopic derivation of a $\mathrm{HOH}$, together with the analysis of the validity of existing theories, is still a point in question. It has been shown [146] that for the case of Maxwellian molecules, whereas Navier-Stokes approximation yields equations which are stable against small perturbations, this is not the case when are introduced Burnett contributions to the equations. It follows that small perturbations to the solutions, which are periodic in the space variable with a wavelength smaller than a critical length, are exponentially unstable. This fact has been called Bobylev's instability. More recently, Garcia-Colin and collaborators [147] have extended Bobylev's analysis for the case of any interaction potential, and have correctly argued that one can interpret the fact as to give a bound for a Knudsen number above which the Burnett equations are not valid. Moreover, Karlin [148] reconsidered the question looking for exact solutions to simplified models.

Furthermore, inclusion of nonlinearity in the theory, in a Nonlinear Higher-Order Thermo-Hydrodynamics (NLHOTH for short and meaning thermal physics of fluid continua), leads to additional possible singularities, called hydrodynamic singularities, as, for example, those described in Refs. $[149,150]$. A satisfactory construction of a NLHOTH is highly desirable for covering a large class of hydrodynamic situations obtaining an understanding of the physics involved from the microscopic level, and, as already noticed, in the last instance gaining insights into technological and industrial processes as in, for instance, hydraulic engineering, food engineering, soft-matter engineering, and so on, which have an associated economic interest.

We recall what was said before that nowadays two approaches appear to be the most favorable for providing very satisfactory methods to deal with hydrodynamics within an ample scope of nonequilibrium conditions. They are Nonequilibrium Molecular Dynamics (NMD) [31-37] and the kinetic theory based on the far reaching generalization of Gibbs' ensemble formalism, the Nonequilibrium Statistical Ensemble Formalism (NESEF for short) [1,3,4,151,152] presented in sections III and IV. NMD is a computational method created for modeling physical system at the micro- scopic level, being a good technique to study the molecular behavior of several physical processes.Together with the so-called Monte Carlo method are part of what is known as numeric simulation methods [32], or Computational Physics (which is to be added to the old classification in specialities of Experimental and Theoretical Physics) [33].

An extensive derivation of a NLHOTH on the basis of the kinetic theory founded on NESEF, quite appropriate to deal with systems in far-from-equilibrium conditions involving the development of ultrafast relaxation processes, and displaying nonlinear behavior leading, eventually, to instabilities and synergetic self-organization [153] is presented in Refs. $[3,71,87,133,134]$.

It may be noticed that the formalism can be extended to deal with the so-called non-conventional or "anomalous" hydrodynamics which is associated to disordered media [154], consisting in systems showing a complex structure of a fractal-like (self-affine on average) characteristics, whose range of applicability and of physical interest is large. Fall on this problem the case of the distinctive behavior of polyatomic structures such as colloidal particles, surfactant micelles, and polymer and biopolymer (as DNA) molecules in a liquid, which are classical examples of what is presently referred to as soft condensed matter $[24,155]$. One particular case of apparently unusual behavior is the one associated to hydrodynamic motion leading to a so-called "anomalous" diffusion, better called non-Fickian diffusion, described by a time evolution following a kind of fractional-power law [156]: The question is considered in Section XII.

The generalized higher-order hydrodynamics built in the framework of NESEF-kinetic theory is characterized by the microdynamical variables consisting of the density of particles, $\hat{n}(\mathbf{r})$, the density of energy, $\hat{h}(\mathbf{r})$, and their fluxes of all order, i.e., the tensors of rank $r, \hat{I}_{n}^{[r]}(\mathbf{r})$ and $\hat{I}_{h}^{[r]}(\mathbf{r})$, with $r=1,2, \ldots$, given by (see Eqs. (20) to (22) in section III)

$$
\begin{gathered}
\hat{n}(\mathbf{r})=\int d^{3} p \hat{n}_{1}(\mathbf{r}, \mathbf{p}), \\
\hat{h}(\mathbf{r})=\int d^{3} p \frac{p^{2}}{2 m} \hat{n}_{1}(\mathbf{r}, \mathbf{p}), \\
\hat{\mathbf{I}}_{n}(\mathbf{r})=\int d^{3} p \frac{\mathbf{p}}{m} \hat{n}_{1}(\mathbf{r}, \mathbf{p}), \\
\hat{\mathbf{I}}_{h}(\mathbf{r})=\int d^{3} p \frac{\mathbf{p}}{m} \frac{p^{2}}{2 m} \hat{n}_{1}(\mathbf{r}, \mathbf{p}), \\
\hat{I}_{n}^{[r]}(\mathbf{r})=\int d^{3} p u^{[r]}(\mathbf{p}) \hat{n}(\mathbf{r}, \mathbf{p}), \\
\hat{I}_{h}^{[r]}(\mathbf{r})=\int d^{3} p u^{[r]}(\mathbf{p}) \frac{p^{2}}{2 m} \hat{n}_{1}(\mathbf{r}, \mathbf{p}),
\end{gathered}
$$

where

$$
u^{[r]}(\mathbf{p})=\left[\frac{\mathbf{p}}{m} \cdots(r-\text { times }) \cdots \frac{\mathbf{p}}{m}\right]
$$


which is the tensorial product of $r$ vectors $\mathbf{p} / m$, defining a $r$-rank tensor (it has the dimensions of velocity to the power $r)$.

The average over the nonequilibrium ensemble of the quantities above provides the set of macrovariables

$$
\left\{n(\mathbf{r}, t), h(\mathbf{r}, t), \mathbf{I}_{n}(\mathbf{r}, t), \mathbf{I}_{h}(\mathbf{r}, t),\left\{I_{n}^{[r]}(\mathbf{r}, t)\right\},\left\{I_{h}^{[r]}(\mathbf{r}, t)\right\}\right\},
$$

with $r=2,3, \ldots$ which define the generalized hydrodynamics. It can be noticed that those associated to the energy are contained in those associated to the movement of matter: Beginning with $I_{n}^{[2]}$ the contraction of the first two indexes provide the expression for the energy; such contraction in $I_{n}^{[3]}$ results in the flux of energy (heat current), and so on. Therefore, in what follows we deal only with the macrovariables of the former (index $n$ ), that is to say

$$
I_{n}^{[r]}(\mathbf{r}, t)=\int d^{3} p u^{[r]}(\mathbf{p}) f_{1}(\mathbf{r}, \mathbf{p} ; t),
$$

with $r=0$ providing the density $n(\mathbf{r}, t), r=1$ the vectorial flux $\mathbf{I}_{n}(\mathbf{r}, t)$, and $r \geqslant 2$, the higher-order tensorial fluxes, and where $f_{1}(\mathbf{r}, \mathbf{p} ; t)$ is NESEF-Boltzmann single-particle distribution function in space $(\mathbf{r}, \mathbf{p})$ of Eq. (45) in Section IV. Moreover, it can be shown that we can write for the flux

$$
\mathbf{I}_{n}(\mathbf{r}, t)=n(\mathbf{r}, t) \mathbf{v}(\mathbf{r}, t),
$$

where $\mathbf{v}(\mathbf{r}, t)$ is the field of barycentric velocity, and for the second-order flux that

$$
m I_{n}^{[2]}(\mathbf{r}, t)=P^{[2]}(\mathbf{r}, t)+m[\mathbf{v}(\mathbf{r}, t) \mathbf{v}(\mathbf{r}, t)],
$$

where $P^{[2]}$ is the pressure tensor and the last term on the right is the convective pressure tensor.

The equations of evolution of these macrovariables provide us with the hydrodynamic equations in this NLHOH. They are

$$
\begin{gathered}
\frac{\partial}{\partial t} I_{n}^{[r]}(\mathbf{r}, t)=\int d^{3} p u^{[r]}(\mathbf{p}) \frac{\partial}{\partial t} f_{1}(\mathbf{r}, \mathbf{p} ; t), \\
\frac{\partial}{\partial t} I_{h}^{[r]}(\mathbf{r}, t)=\int d^{3} p \frac{p^{2}}{2 m} u^{[r]}(\mathbf{p}) \frac{\partial}{\partial t} f_{1}(\mathbf{r}, \mathbf{p} ; t),
\end{gathered}
$$

that is, are all determined by the equation of evolution of the single-particle distribution $f_{1}$, which is given in Eq. (45). Performing the lengthy calculations involved one finally arrives at the general evolution equations $(\ell=0,1,2, \ldots)$

$$
\begin{gathered}
\frac{\partial}{\partial t} I_{n}^{[\ell]}(\mathbf{r}, t)+\nabla \cdot I_{n}^{[\ell+1]}(\mathbf{r}, t)= \\
=-\frac{1}{m} \sum_{s=1}^{\ell}\left\{\tau(1, s)\left[\mathbf{F}(\mathbf{r}, t) \otimes I_{n}^{[\ell-1]}(\mathbf{r}, t)\right]+\right. \\
\left.+\theta_{\ell}^{-1} I_{n}^{[\ell]}(\mathbf{r}, t)\right\}+a_{L o} \sum_{s=1}^{\ell}\left\{\tau(1, s)\left[\nabla I_{n}^{[\ell-1]}(\mathbf{r}, t)\right]+\right. \\
\left.+2 \ell a_{L 1} \nabla \cdot I_{n}^{[\ell+1]}(\mathbf{r}, t)+S_{n}^{[\ell]}(\mathbf{r}, t)\right\},
\end{gathered}
$$

where we have put into evidence the presence of the fluxes neighboring the one of order $\ell$, namely $I^{[\ell-1]}$ and $I^{[\ell+1]}$. The symbol $\tau(1, s)$ is an operator that performs the transposition of indexes 1 and $s$ of the following tensor. And $\mathbf{F}(\mathbf{r}, t)$ is minus the force term of Eq. (47), after deleting $\mathbf{B}_{1}(\mathbf{p})$. The $\ell$-rank tensor $S_{n}^{[\ell]}(\mathbf{r}, t)$ contains the contributions involving all the other fluxes, the thermo-striction effects, and the action of the external sources, having a pretty cumbersome expression. Moreover,

$$
\theta_{\ell}^{-1}=-\ell\left[\left|a_{\tau 0}\right|+(\ell-1)\left|b_{\tau 1}\right|\right]
$$

is playing the role of a Maxwell-characteristic time for the $\ell$-th flux, and the several kinetic coefficients $a$ 's and $b$ 's are given elsewhere [85]. These characteristic times follow a hierarchy of values; in fact, we do have that

$$
\frac{\theta_{\ell+1}}{\theta_{\ell}}=\frac{\ell}{\ell+1} \frac{5 \zeta+\ell-1}{5 \zeta+\ell},
$$

for $\ell=1,2,3, \ldots$, where $\zeta$ is a quantity depending on the ratio of masses $m$ and $M$, such that it is verified the succession of decreasing values

$$
\theta_{1}>\theta_{2}>\theta_{3}>\ldots>\theta_{\ell}>\theta_{\ell+1}>\ldots,
$$

which can be considered to represent Boguliubov's hierarchy of characteristic times $[56,58]$ in generalized hydrodynamics.

Evidently, Eq. (75) consists of a set of nonlinear integrodifferential equations coupling all the densities and fluxes, being of unmanegeable proportions. However, in practical situations enters into play Boguliubov's principle of correlation weakening $[54,58]$ and the accompanied hierarchy of relaxation times, which in this case, as noticed above, consists of the hierarchy of Eq. (78). Hence resorting to Boguliubov's principle it is possible to introduce a contraction in the set of hydrodynamic evolution equations $[3,4]$. Thus, taking only the densities (accompanied with constitutive equation for their first fluxes) constitutes a NLHOH of order zero, and it leads to the standard Fick and Fourier diffusion equations. Taking the densities and their first fluxes, we do have a NLHOH of order 1, leading to the Maxwell-Cattaneo evolution equations for the densities. Taking the densities and their first and second fluxes, we have a NLHOH of order 2, leading to the to evolution equations of the densities of third order in time, and so on and so forth $[3,4,54,58]$.

\section{COHERENT STATES AND NONEQUILIBRIUM BOSE-EINSTEIN-LIKE CONDENSATIONS}

It has been noticed by D. Snoke [76] that Bose-Einstein condensations occurs when many particles enter into the same, coherent quantum state, and is now claimed to occurs in various systems of "quasiparticles" in solids. But is it the right term to use here?

$\mathrm{He}$ is talking about the particular phenomena that arise in bosons systems in nonequilibrium conditions, as those mentioned below. He argues that in that cases, rather than haggling over the exact meaning of the term Bose-Einstein condensation, we can adopt a more general concept that allows us to use the same language to talk about many systems. This concept is the spontaneous emergence of coherence. However, it may be noticed that such situations belong 
to a, say, discipline defining and studying synergetic selforganization initiated around half century ago by Hermann Haken [8] and Ilya Prigogine [9]. It precisely covers these, so-called, nonequilibrium phase transitions arising out of order through fluctuations (sometimes referred to as order out of chaos [157]), also involving the so-called complex behavior.

Complex systems is the name for short of systems displaying some kind or other of complex behavior, which can emerge when the system is sufficiently far-from equilibrium and governed by a nonlinear macroscopic kinetics. As noticed above it is present in systems of bosons when under such conditions. We can cite:

- "Bose-Einstein-like condensation" of LO phonons (Fröhlich effect) in biopolymers, generated by dark excitation, which is accompanied by the emission of solitons of the Schroedinger-Davydov type, possessing a very long half life [158] (of interest in Physiology and Neurology).

- "Bose-Einstein-like condensation" of acoustic phonons in biological material, generated by sonic excitation, which is accompanied, as in the previous case, by the emission of solitons of the Schroedinger-Davydov type with a very long life time [159] (of interest in Medicine).

- "Bose-Einstein-like condensation" and propagation of Schroedinger-Davydov solitons of very long life time of excitons: the case of the "excitoner" ("laser" of coherent excitons) [160].

- "Bose-Einstein-like condensation" of acoustic phonons, generated by drifting-electron excitation ("saser" or "laser" of acoustic phonons) [161,162].

- "Bose-Einstein-like condensation" of LO phonons generated by drifting-electron excitation ("laser" of LO phonons) [163].

- Emission of LO phonons in so called coherent states by electromagnetic excitation, of interest in semiconductor devices [164].

As already noticed, the case of systems far from equilibrium encompasses an area of particular interest, namely systems that may display the so-called complex behavior, which can arise in systems whose evolution is governed by nonlinear macroscopic kinetic laws (see for example [8-11]). Certainly, NESEF has a relevant role in the realm of Complexity Theory and a fundamental place in the expanding domain of nonlinear physics. Nonlinearity has a fundamental role in determining complex behavior in open systems far-away-from equilibrium. In many cases it shall lead to the emergence of self-organized synergetic behavior at the macroscopic level, in the form of the so-called Prigogine's dissipative structures [9,10,165-167]. Dissipation is not in such circumstances a source of decay but has a constructive role, maybe including the emergence of life, natural evolution, and the astounding functioning of living systems. This is the question of order out of chaos in complex systems, i.e. synergetic processes leading to self-organization in open systems far-fromequilibrium, when, as noticed, are governed by nonlinear macroscopic kinetic laws.
Such kind of complex behavior can arise in solid state matter, and we briefly summarize a few examples in semiconductors, polymers, and biological systems. In Refs. [158$164,168-173$ ] is given an overview of the role of the irreversible thermodynamics based on NESEF in dealing with biosystems. On the question of the relation of Biology and Physics we may say that the sometimes mentioned gap between both disciplines has been slowly shrinking along recent decades. The old difficulty with this sought after connection, which resided into looking exclusively at the microscopicmechanicist level of Physics, disappears when one begins to consider the macroscopic level of Physics. An important role is played by the thermodynamics of nonlinear nonequilibrium open dissipative systems, as it is the Informational-Statistical Thermodynamics, build on the basis of the NESEF [13-16], already mentioned in Section III. NESEF has been applied to the determination of the complex behavior that may result in biopolymers, namely, Fröhlich-Bose-Einstein-like condensation and propagation of Davidov's solitary-wave-like excitations, which are of relevance in Bioenergetics. The question of the lifetime of the latter at physiological conditions was discussed, and the case was illustrated in a comparison with experiments performed in the case of an organic molecular polymer, as it is briefly described below, with no detail being presented which can be obtained in the references that are cited.

The interesting phenomenon of the so-called FröhlichBose-Einstein-like condensation [174] - as viewed from the NESEF angle - has been considered in several articles. Mesquita et al. [158] have considered the nonequilibrium and dissipative evolution, and the steady state of the population of vibrational polar modes in a chain of biomolecules. These polar modes are excited through a coupling with a pumping source of metabolic energy and are in anharmonic interaction with an elastic continuum. Groups of polar modes are coupled in this way through nonlinear terms in the kinetic equations. This nonlinearity is shown to be the source of an unexpected phenomenon characterizing complex behavior in this kind of system: after a threshold of intensity of the pumping source is achieved, polar modes with the lowest frequencies increase enormously their population in a way reminiscent of a Bose-Einstein condensation (Fröhlich effect). The transient time for the steady-state condensate to follow is very short (picosecond time scale) and the condensation appears even for weak values of the anharmonic coupling strength responsible for its occurrence. Further, it seemingly requires accessible levels of pumping power of metabolic energy in order to be produced and sustained.

The question was further pursued in [158,168-172], where the above mentioned Fröhlich effect - consisting, as noticed, of a coherent behavior of boson-like excitations in biological and molecular polymers - is fully derived and analyzed in terms of NESEF. It was shown that when double (or multiple) processes of excitation of the boson system are possible there follows a positive-feedback phenomenon that greatly favors and enhances the effect [174].

An alternative possibility for Fröhlich condensation to follow due to higher-order relaxation processes mediated by carriers - as for example in proteins -, was considered by L. Lauck et al. [173], who took into account the above model of a biological system consisting of a long chain of 
proteins possessing polar modes of vibration, and where energy is pumped through metabolic processes. It was considered the effect produced by free electrons that are usually present as hole carriers in proteins with electron-donor molecules. NESEF was used for the derivation of the kinetic equations and introducing the non-linearities due to interactions of the polar vibrations with the carriers and with the thermal bath. These non-linearities arising out of higher order relaxation processes lead to the emergence of the Fröhlich effect in the polar modes, i.e., as noticed, the occurrence of a peculiar (nonequilibrium) Bose-Einstein-like condensation. It also points to an instability of the system that seems to be followed by a morphological transformation in the form of a spatially ordered dissipative structure.

Another phenomenon, which is closely related to this phenomenon of Fröhlich-Bose-Einstein condensation in a nonequilibrium phase (in the sense that it is a result of the same many-body system microdynamics, being governed by the same Hamiltonian operator and nonlinear macroscopic kinetic effects), consists in the propagation of solitary waves in several systems. Solitary waves are ubiquitous and of relevance in a number of situations in applied science [175]. They play a fundamental role in the case of conducting polymers (for use in electric car batteries, microcircuits, etc.) [176-179]. In biological systems, the so-called SchrödingerDavydov's solitary waves [180] may have a relevant role; they have been considered within NESEF in [181], where it was analyzed Davydov's biophysical model in the context of NESEF-based nonequilibrium statistical thermodynamics. It was shown that excitations of the Davydov-soliton type that can propagate in the system, which are strongly dampened in near-equilibrium conditions, become near dissipation-free in the Fröhlich-Bose-Einstein condensate and that this occurs after a certain threshold of pumped metabolic energy is reached. This implies in the propagation of excitations at long distances in such biosystems. The question have been further analyzed and extended in [182], and in [183] is considered the case of the organic polymer acetanilide, which mimics some aspects of certain biopolymers and has been subjected to extensive experimental analysis.

We close this section mentioning some applications of NESEF to the study of complex behavior - of the type of formation of Prigogine's dissipative structures - in far-fromequilibrium many-body systems. Morphological ordering of the reaction-diffusion Turing type is considered in [184], where is analyzed the dissipative thermodynamic regime of an electron system in bulk matter under the action of an external source of energy, which generate electron-hole pairs with a nonequilibrium distribution in energy space. It was shown that with increasing values of the source power (furthering the distance from equilibrium), and strictly in the case of a $p$-doped material, the carrier system displays complex behavior characterized by undergoing a succession of transitions between synergetically self-organized dissipative structures. The sequence goes from the homogeneous steady state (or stochastic thermal chaos), to sinusoidal spatial deviations (morphological ordering), to intricate ordered states (subharmonic bifurcations), and finally to a situation resembling turbulent-like-chaos (a multi- periodic spatial organization). The phenomenon may arise, for example, in semiconductor systems, molecular polymers, and protein molecular chains in biosystems.

Moreover, in [185] is considered the nonlinear macroscopic kinetics of evolution of the carrier system in the photoinjected plasma in semiconductors under the action of constant illumination with ultraviolet light. It was shown that the spatially homogeneous steady-state becomes unstable, and a charge-density wave emerges after a critical intensity of the incident radiation is achieved. For intensities beyond this critical threshold an increasing number of modes provide further contributions (subharmonics) to the space inhomogeneity, leading the system to display chaotic-like behavior, as it is in [184]. It was shown that this phenomenon can only follow in doped $p$-type materials as semiconductors and some molecular and biological polymers, the latter when under dark biochemical excitation.

\section{THERMO-STATISTICS OF COMPLEX STRUCTURED SYSTEMS AND NESEF}

More than twenty years ago Montroll and Shlesinger wrote that in the world of the investigation of complex phenomena that requires statistical modelling and interpretation several competing styles have been emerging, each with its own champions [186]. What is at play consists in that in the study of certain physico-chemical systems we may face difficulties when handling situations involving fractal-like structures, correlations (spatial and temporal) with some type of scaling, turbulent or chaotic motion, small size (nanometric scale) systems with eventually a low number of degrees of freedom, and so on: It is in these situations that we are faced with the existence of so-called hidden constraints to which we do not have access. The interest on the study of such kind of complex physical systems has been recently enhanced as a consequence that they are part of electronic and opto-electronic devices of the nowadays advanced technologies, and also in technological/industrial areas involving the use of disordered systems, polymeric solutions and materials, ion-conducting glasses, the case of microbatteries, and others. The question involves the fact that the researcher faces difficulties in satisfying Fisher's Criteria of Efficiency and Sufficiency [187] in the conventional approach to the well established, physically and logically sound Boltzmann-Gibbs statistics ("The criterion of efficiency is satisfied by those statistics which, derived from large samples, tend to a normal distribution with the least possible standard deviation" and, "A statistics satisfies the criterion of sufficiency when no other statistics which can be calculated from the same sample provides any additional information as the value of the parameter to be estimated", which for the purposes of statistical mechanics is to be taken as the existence of an incomplete description of the physical situation in hands [188]). In Statistical Mechanics the question typically consists in that one needs to confront some impairment on how to correctly include the presence of large fluctuations (and eventually higher-order variances) and to account for the relevant and proper characteristics of the system - implying in lack of efficiency and sufficiency, respectively. As a consequence, in an attempt to assuage these difficulties, and thus allowing to improve the predictions, one may resort to statistical approaches other than the consistent canonical one of Boltzmann-Gibbs ensemble formalism. 
Among existing approaches we can mention:

1. The one used by P. T. Landsberg showing that functional properties of the (informational) entropies give in fact origin to different types of unusual thermostatistics, and rise the question of how to select a "proper" one, that is, are some better than others? [189];

2. For decades it has been in use Lèvy Statistics, introducing modified non-Gaussian distributions which has been applied to a variety of problems, and recently it had a revival with its application to the study of chaotic dynamics (see for example Ref. [186,190,191]);

3. The approach of W. Ebeling who has addressed the question of the statistical treatment of a class of systems that are in some sense "anomalous": They contain those in nature and society which are determined by its total history. Usually the given examples are the evolution of the Universe and of our planet, phenomena at the biological, ecological, climatic, social levels, etc. The approach consists into introducing conditional probabilities in the context of BoltzmannGibbs formalism in Shannon-Jaynes approach, leading to a thermo-statistics appropriate for describing complex processes with long-ranging memory and including correlations [192-194].

4. So-called Superstatistics developed by C. Beck and E. G. D. Cohen for non-equilibrium systems with complex dynamics in stationary states with large fluctuations on long-time scales [195];

5. The generally called Non-Extensive Thermo-Statistics, based on Havrda-Charvat approach [196], which applied to several cases is described in the Conference Proceedings of Ref. [197];

6. Renyi approach [198] has been introduced in the scientific literature, as noticed in Ref. [199], with, for example, P. Grassberger and I. Procaccia [200,201] using it in a modified form as a valuable method for characterizing experimental chaotic signals. Recently P. Jizba and T. Arimitzu [202] have presented an extensive analysis of it in a paper called "The world according to Renyi", where they show that making extremal Shannon's entropy on a multifractal is equivalent to directly making extremal Renyi's entropy without invoking the multifractal structure explicitly;

7. Sharma-Mittal approach [203] or better to say a variation of it (called Kappa or Deformational Statistics), was used by V. M. Vasyliunas in problems of astrophysical plasma [204] and by G. Kanadiakis in the case of relativistic systems [205].

We recall that in Statistical Mechanics the probability distribution (statistical operator), usually derived from heuristic arguments, can also be derived from an extremum-principle formalism once is made connection with Information Theory $[3,206,207]$. It consists into making a maximum, subjected to certain constraints, a functional (superoperator) of the probability distribution (statistical operator). Such quantity, first introduced in Shannon's Theory of Communication [51-53], can be referred-to as measure of uncertainty of information. It has also been called statistical measure and entropy, with the understanding that it is information-theoretic entropy. It is worth to emphasize - in view of some confusion that has recently pervaded the scientific literature - that the different possible information-theoretic entropies are not to be interpreted as the thermodynamic entropy of the physical system: the renowned theoretical statistician R. T. Cox has noticed that the meaning of such entropies is not the same in all respects as that of anything which has a familiar name in common use, and it is therefore impossible to give a simple verbal description of it, which is, at the same time, an accurate definition [208]. Moreover, E. T. Jaynes has also commented that it is an unfortunate terminology, and a major occupational disease in that there exists a persistent failure in discerning between the informational entropy, which is a property of any probability distribution, and the experimental entropy of thermodynamics which is instead a property of a thermodynamic state: Many research papers are flawed fatally by the authors' failure to distinguish between these entirely different things, and in consequence providing nonsense results [209].

Gibbs-Boltzmann-Shannon information-theoretic entropy (Kullback-Leibler measure in Information Theory [210]) is given by

$$
S_{G B S}(t)=-\operatorname{Tr}\{\rho(t) \ln \rho(t)\}
$$

with $\rho(t)$ being the statistical operator for the corresponding Gibbs' ensemble. The derivation of the statistical operator $\rho(t)$ has been done using various consistency arguments: see for example the review article by Balian and Balazs in Ref. [211]. Among them we can highlight the one based on heuristic arguments (as it is usual in the textbooks for the case of equilibrium, and for non-equilibrium systems see for example [212,213]), and the one using an extremum-principle approach consisting in maximizing $S_{G B S}(t)$ subjected to constraints [3,206] (the so-called MaxEnt formalism).

As emphasized by Balian and Balazs [211], this GBS definition relies on an invariance property, the invariance under unitary transformations, and it is the only function of the density operator which satisfies the sub-additivity inequality for compound systems [189,214,215]. Moreover, as already noticed, there is no reason for abandoning the informationtheoretic Shannon (Kullback-Leibler) entropy, which is the only consistent probabilistic measure of information.

On the other hand, the Havrda-Charvat structural $\alpha$ entropy and others of the like (in principle infinitely-many) are measures of uncertainty of information which have been used in different areas of knowledge since the mid nineties (see Ref. [216]) and the one due to Paul Lèvy since the 1930's [186]. In Physics, as noticed, they can be used as an auxiliary formalism to make predictions when dealing with systems displaying some kind or other of complex behavior, in which there are hidden constraints (relevant to characterize the state - either macroscopic and/or microscopic - of the system), that is, constraints to which we do not have access.

We briefly describe these alternative approaches, and its use within the extremum-principle formalism MaxEntNESEF (for Maximization of Informational Entropy in the Non-Equilibrium Statistical Ensemble Formalism 
$[1,3,45,50])$, for deriving non-conventional non-equilibrium statistical-mechanical distributions.

We begin noticing that Statistical Mechanics of many-body systems has a long and successful history; see Section II. The introduction of the concept of probability in physics originated mainly from the fundamental essay of Laplace [217], who incorporated and extended some earlier seminal ideas (see an historical review in Ref. [218]). As well known, Statistical Mechanics attained the status of a well established discipline at the hands of Maxwell, Boltzmann, Gibbs, and others, and went through some steps related to changes, not in its fundamental structure, but just on the substrate provided by microscopic mechanics. Its application to the case of systems in equilibrium proceeded rapidly and with exceptional success: equilibrium statistical mechanics gave - starting from the microscopic level - foundations to Thermostatics, and the possibility to build a Response Function Theory. Applications to non-equilibrium systems began, mainly, with the case of local equilibrium in the linear regime following the pioneering work of Lars Onsager [130,219].

For systems arbitrarily deviated from equilibrium and governed by nonlinear macroscopic kinetic laws, the derivation of an ensemble-like formalism proceeded at a slower pace than in the case of equilibrium, and somewhat cautiously, with a long list of distinguished scientists contributing to such development. It can be noticed that Statistical Mechanics gained in the nineteen fifties an alternative approach sustained on the bases of Information Theory [61,206,218,220223]: It invoked the ideas of Information Theory accompanied with ideas of scientific inference [224,225], and an extremum-principle formalism (the latter being Jaynes' principle of maximization of informational uncertainty - the so called informational-entropy as notice before - and called MaxEnt for short), compounding from such point of view a theory dubbed Predictive Statistical Mechanics. It should be noticed that this is not a new paradigm in Statistical Physics, but a quite useful and practical variational method which codifies the derivation of probability distributions, which are usually obtained by either heuristic approaches or projection operator techniques. It is particularly advantageous for building non-equilibrium statistical ensembles allowing for the systematization of the relevant work on the subject that renowned scientists provided along the past century as describe in the book of Ref. [3] and commented upon in Sections II and III.

Moreover, it has been noticed that MaxEnt is not a physical principle in the proper sense, and should be carefully distinguished from the "entropy maximum principle" of thermodynamics: The latter is not a rule of inference but a condition of thermodynamic equilibrium [225].

MaxEnt, apparently first proposed by W. Elssasser [60], was analyzed in depth, largely systematized and extended by E. T. Jaynes, who proposed it as an extension of the classical principle of insufficient reason. It has been claimed that MaxEnt is single out as a unique method of statistical inference that agree with certain compelling consistency requirements: The point has been critically reviewed by J. Uffink [226]. Moreover, Rolf Landauer [227] has argued that "advocacy of MaxEnt is perpertuated by selective decision making in the generation of papers [...] MaxEnt is likely to be sound, but often it is dreadfully difficult to understand what the constraints are". Mario Bunge stated [228] that "when confronted with a random or seemingly random process, one attempts to build a probabilistic model that could be tested against empirical data; no randomness, no probability. Moreover, as Poincaré pointed out long ago, talk of probability involves some knowledge; it is not a substitute for ignorance (and Bunge adds, not correctly in what refers to the statistical mechanics we are discussing here, that) this is not how the Bayesian or personalists view the matter: when confronted with ignorance or uncertainty, they use probability - or rather their own version of it. This allows them to assign prior probabilities to facts and propositions in an arbitrary manner [again, this is not the case in MaxEnt-NESEF] - which is a way of passing off mere intuition, hunch, or guess for scientific hypothesis [...]; it is all a game of belief rather than knowledge".

Sometimes arguments against MaxEnt in terms of playing dices have been advanced. To this, it must be recalled that the question we are addressing here does not deal with gambling, but with many-body theory. That is, we deal with systems with very many degrees of freedom, and then is necessary to have in mind the distinction between interpretations in terms of microscopic and macroscopic variables.

Concerning the arguments that knowledge arises out of ignorance, this is simply unnecessary confusion coming from a wrong interpretation of, maybe, a sometimes not correct phrasing used by some practitioners of MaxEnt in areas other than Many-Body Physics. Quite to the contrary, the spirit of the formalism is to make use of the restricted knowledge available, but without introducing any spurious one. Quoting Laplace [217], "the curve described by a molecule of air or of vapor is following a rule as certainly as the orbits of the planets: the only difference between the two is our ignorance. Probability is related, in part to this ignorance, in part to our knowledge". Also, as pointed out by Bricmont [229], "the part due to our ignorance" is simply that we use probabilistic reasoning. If we were omniscient, it would not be needed (but the averages would remain what they are, of course). The part "due to our knowledge" is what makes reasoning work [...]. But this is the way things are: our knowledge is incomplete, and we have to live with that. Nevertheless, probabilistic reasoning is extraordinarily successful in practice, but, when it works, this is due to our (partial) knowledge. It would be wrong to attribute any constructive role to our ignorance. And it is also erroneous to assume that the system must be somehow indeterminate when we apply probabilistic reasoning to it." (see also Ref. [230]).

It has been noticed that to derive the behavior of the macroscopic state of the system from partial knowledge has been already present in the brilliant original work of Gibbs. Werner Heisenberg wrote [89] "Gibbs was the first to introduce a physical concept which can only be applied to an object when our knowledge of the object is incomplete". Furthermore, it can be considered that the dismissal of a theoretical approach in Physics cannot (and should not) be done on the basis of general verbal arguments, which may or may not be sensible, but which need be strongly fundamented on the scientific method. In other words, the merits, or rather demerits, of a theory reside in establishing its domain of validity (see for example Refs. [87] and [90]), when tested against the experimental results it predicts. This point has recently been forcefully stressed by Stephen Hawkings [231]. On the 
particular case of the extremum-principle approach in Jaynes style (as an alternative way [212] to, e.g., the heuristic one [4] for building NESEF) Lawrence Sklar has summarized that Jaynes firstly suggested that equilibrium statistical mechanics can be viewed as a special case of the general program of systematic inductive reasoning, and that, from this point of view, the probability distributions introduced into statistical mechanics have their bases not so much in an empirical investigation of occurrences in the world, but, instead in a general procedure for determining appropriate a priori subjective probabilities in a systematic way $[151,232]$.

Additional analyses are present in the work of B. C. van Fraassen [233-235], where it is discussed the possibility of alternative rules of construction of generalized (informationtheoretic) entropy expressions containing a free continuous parameter (so-called Renyi entropies), that is, of the type of those considered here: Uffink noticed that it seems that more research would be needed to assess their performance in concrete cases and in general. Moreover, in an article properly titled Entropies Galore! [189], Peter Landsberg have called the attention to the fact that (see Ref. [216]) very-many of these information-theoretic entropies can be proposed; and adds the question of how to select a "proper" one, or, "Are some better than others?" No clear "selection rule" can be forwarded. At this point we reiterate our comment that a most used is Renyi's information-theoretic entropy (ITE) [198], which appears as the most convenient one to deal with multifractal complex systems [202]. It can also be noticed that HavrdaCharvat ITE [196] (or structural $\alpha$-entropy, recently renamed $q$-entropy) provides the same probability distribution than the one that follows from Renyi ITE (they differ in the definition of the Lagrange multipliers that the extremum-principle approach introduces, and it can be noticed that Renyi ITE avoids difficulties associated to the other). It can be mentioned that they have been used in a particular problem (with "hidden constraints") of marketing of different brands of toilet soup [236], producing the same result [216], as it should.

The informational-based approach has been quite successful in its application to the cases of equilibrium and near equilibrium conditions [212,213,221,237,238], and in the last decades has been, and is being, also applied to the construction of a generalized ensemble theory for systems arbitrarily away from equilibrium $[3,14,15,45,49,239]$. NESEF provides mechanical-statistical foundations to irreversible thermodynamics (in the form of Informational Statistical Thermodynamics (ITE for short [13-16]), a nonlinear quantum kinetic theory $[1,3,45,59,67-69,240]$ and a response function theory [3,241] of a large scope for dealing with many-body systems arbitrarily away from equilibrium. NESEF has been applied with success to the study of a number of non-equilibrium situations in the physics of semiconductors (see for example the review articles of Ref. [78]) and polymers [183], as well as to studies of complex behavior of boson systems in, for example, biopolymers (e.g. Refs. [158-160,168-172,181-184]) phonon systems [162-164], and magnon systems [161]. It can also be noticed that the NESEF-based nonlinear quantum kinetic theory provides, as particular limiting cases, farreaching generalizations of Boltzmann and Mori equations [67-69,71] and a Higher-Order Hydrodynamics, linear and nonlinear $[85,133,134]$.

As already noticed, NESEF is built on the bases of heuris- tic standard arguments (see for example [4], or within the scope of the extremum-principle method based on the maximization of the information-theoretic-entropy of ShannonJaynes approach in Boltzmann-Gibbs statistics [3], that is, the average of minus the logarithm of the time-dependent i.e. depending on the irreversible evolution of the macroscopic state of the system - non-equilibrium statistical operator. We again emphasize that information-theoretic-entropy is in fact the quantity of uncertainty of information, and has the role of a generating functional for the derivation of probability distributions (for tackling problems in Communication Theory, Physics, Mathematical Economics, and so on). There is one and only one situation when Shannon-Jaynes informational-entropy coincides with the physical entropy of Clausius in thermodynamics, namely, the case of strict equilibrium [188,242-244].

We recall that the extremum-principle approach produces the well established equilibrium statistical mechanics, and is providing a satisfactory formalism for describing nonequilibrium systems in a most general form. Such conventional (or canonical) approach in Boltzmann-Gibbs Statistical Mechanics allows for a proper description of the physics of condensed matter, but in some kind of situations, for example, involving nanometric-scale systems with some type or other of fractal-like structures or systems with long-range space correlations, or particular long-time correlations, it becomes difficult to apply because of a deficiency in the proper knowledge of the characterization of the states of the system in the problem one is considering (at either the microscopic, macroscopic or mesoscopic levels): In other words we are then studying a system facing the difficulty of being present hidden constraints to which we do not have access.

We have already called the attention to the classical and fundamental paper of 1922 [187] by R. A. Fisher, titled "On the Mathematical Foundations of Theoretical Statistics", where the basic criteria that a statistics should satisfy in order to provide valuable results are presented. We reiterate that in present day Statistical Mechanics in Physics two of them are of major relevance, namely the Criterion of Efficiency and the Criterion of Sufficiency already described at the beginning of this section. This is so because of particular constraints that are present, for example, in physical situations involving small systems where, on the one hand, the number of degrees of freedom entering in the statistics may be small and, on the other hand, structures, including boundary conditions of a fractal-like character strongly influence the properties of the system. Such facts make it difficult to introduce sufficient information for deriving a proper Boltzmann-Gibbs probability distribution, and we may mention the examples of nanotechnology, nanobiophysics, quantum dots and nanometric heterostructures in semiconductor devices, one-molecule transistors, fractals-electrodes in microbatteries, and so on. Other case when the sufficiency criterion is difficult to satisfy is the one of large systems of fluids whose hydrodynamic motion is beyond the domain of validity of the classical standard approach. It is then required the use of a nonlinear higher-order hydrodynamics, eventually including correlations and other variances (a typical example is the case of turbulent motion) and in, for example, the study of the hydrodynamic motion of complex (average-fractal like) structures in polymer solutions. 
As described in the first part of this Section, a way to improve our capacity to make predictions about the properties and physical characteristic in such kind of situations involving the presence of hidden constraints, consists into resorting to the introduction of modified forms of the informationtheoretic-entropy (that is, others than the consistent one of Shannon), which are built in terms of the available characterization one does have of the system. These informationtheoretic entropies, via MaxEnt, led to the derivation of nonconventional probability distributions (statistical operators), with which it is possible to make predictions on properties and responses of the system, allowing to analyze its expected physical behavior (when constituting a part of a device one can make predictions on its functioning and on eventual modifications for improving its performance). In the process it is introduced a free parameter (one or several), let us call it the infoentropic index, which has the important role of modifying the weight of the contributions of the different dynamical states that are involved in the processes developing in the system under observation: In that way predictions are improved as compared with the conventional ones in the insufficient description.

This alternative approach originated in the decades of the 1950's and 1960's at the hands of theoretical statisticians (a pioneering proposal is due to P. Lèvy in the 1930's), being extensively used in different disciplines (economy, queueing theory, regional and urban planning, nonlinear spectral analysis, and so on). Some approaches were adapted for use in physics leading to what can be dubbed as Non-Conventional Statistical Mechanics (NCSM for short, see also P. T. Landsberg considerations in Refs. [189] and [215]).

A large number of possible information-theoretic entropies can be explored: An infinite family is the one that can be derived from Csiszer's general measure of information-theoretic entropy (see for example [216]). They, being generating functionals of probability distributions, give rise to particular forms of statistics. These information-theoretic entropies are related to a family of so-called statistical measures in a metric space of statistical distributions, when it is provided a distance from the sought-after statistical distribution to a reference distribution: A principle of minimization of this distance (MinxEnt for short) is equivalent to the maximization of the associated infoentropy (MaxEnt) [216]. A complete presentation of the matter is available in Ref. [245].

\section{NONCONVENTIONAL THERMO-HYDRODYNAMICS}

It has been noticed that the question of a so-called nonstandard or "anomalous" hydrodynamics is associated to disordered media [246], consisting in that disordered systems do not follow the classical laws which describe transport in ordered systems, and that this leads to many kinds of "anomalous" physical properties, that is, following unexpected laws differing from the ones of standard hydrodynamics. The range of applicability and of physical interest is enormous. Fall on this problem the case of the distinctive behavior of polyatomic structures such as colloidal particles, surfactant micelles, and polymer molecules in a liquid, which are classical examples of what is presently referred to as soft- condensed matter [24,247]. One particular case of apparently unusual behavior is the so-called "anomalous" diffusion, better called non-Fickian diffusion, described by a time evolution following a kind of fractional-power law. This seems to be connected with the fact that the motion is proceeding in a medium with a fractal-like structure as a result that the growth (manufacture) of samples inevitably produces fractallike structures [23]. This is of relevance for decisions in the technological and industrial-commercial areas in present-day competitive global economy.

As particular examples of the presence of hidden constraints, we may mention here the case of "anomalous" luminescence in quantum wells with fractal-like boundaries in semiconductor heterostructures [248], used for characterizing and controlling the growth in these materials, in a nondestructive testing procedure, and the case of "anomalous" results in cyclic voltammetry experiments involving fractal electrodes in microbatteries which can be interpreted in terms of "anomalous" diffusion of charges in the electrolyte [249], with, in both cases, application of the theory considered here was done. In those references it is presented a full systematic study of these two examples of systems of high technologi$\mathrm{cal} /$ industrial/commercial interest.

As noticed, of particular large relevance in this question are polymers, which are an attractive subject not only for the own scientific interest but also, and importantly, for their technological-industrial-commercial interest. On this we may mention Ref. [250] for a view on general applications, and it is worth calling the attention to the nowadays importance of polymers in Electronics and Photonics [251-257] and the case of conducting polymers for, e.g., more efficient batteries [250-253]. These materials display a wide variety of unusual rheological phenomena, which are of great importance to the synthesis, processing, performance, and end-use characteristics of them. It is considered that probably a majority of rheological research in the last decades has been devoted to the study of polymeric materials [258]. It has been stated that, when under flow, the kinetic effects associated to the latter exert a major restructuring influence on these fluids. Also, the effects of structure on flow can also be relevant, and these and other aspects of soft-matter rheology are still baffling [257,258]. For example, the diffusion behavior of many polymers cannot be adequately described by the usual Fick equation. Generally, this is the case with the socalled glassy polymers which are said to exhibit "anomalous" or "non-Fickian" behavior [156,259]. We can also include in this class of materials the case of micelles. In those cases it has recently surged the question of how flow (advective motion, particularly under shear flow [260]) affects the system. It is recognized that it is vital to control the flow of polymers and surfactant micelles in many situations, including the production of food, derivatives of petroleum, and household goods, and in various applications in medicine. Particularly, the prediction of the flow of complex fluids, such as solutions of surfactants or polymers, through porous media is of particular interest to various industrial processes: e.g. many oil industry treatment fluids fall in that category [261].

In such cases modelling based on macroscopic approximations tends to fail [261]. A study of the motion of a complex structured fluid in the presence of a forced flow (advective motion) is presented in Ref. [141]. It implies in the in- 
troduction of a particular kind of unification of kinetic and hydrodynamic approaches for the description of processes in active systems, e.g. [45,73,262], described elsewhere [85], adapted for dealing with complex structured systems. In that way it is provided a microscopic-based approach to earlier attempts $[263,264]$ - showing in the process which is the physical interpretation of the hydrodynamic motion developing in the media, and how is obtained the description in terms of "anomalous" hydrodynamic equations - which is used for the purpose of adjusting the description of the motion.

In the case of the hydrodynamic motion in complexstructured fluids mentioned above [141] the "hidden constraints" consist in that the polymer macromolecules differ in number of monomers, and then in mass, (then differences in the translational degrees of motion), in configuration (then differences in internal degrees of motion), and questions of dangling and entanglement. Thus one does not have the complete physical picture of the phenomenon, and then to make predictions obtaining a relatively clear interpretation of the facts involved in the physical processes under way Reny statistics was used. As stated, this is described in Ref. [141], where the use of the theory is fully illustrated, and comparison with experiments done, through its application to the study of surfactant micelles and polymer solutions under flow. Also, for this particular case there is an equivalence of results with those obtained in a description in terms of Lévy flights (see also Ref. [245]).

\section{CONCLUDING REMARKS}

We have presented in this "Feature Article", first, an Introduction with some considerations on the question of the aspects and interrelationships of Science, Technology, Government and Society in the modern world. There it was also called the attention to the "stress" that present day advanced technologies and industrial processes place on the "realm" of Physics, or better to say, to be more specific, on ThermoStatistics of systems driven arbitrarily far from equilibrium. Hence, in Sections II, III and IV it is described the evolution that for that purpose is occurring in Statistical Mechanics of Non-Equilibrium Systems (which is accompanied with a Generalized Irreversible Thermodynamics). Finally, in Sections V to XII we comment on the main physico-chemical processes that are involved in present day advanced technologies and competitive industries of the nowadays "globalized world".

They included, Section VI: Ultrafast Relaxation Processes in Semiconductors; Section VII: Low-Dimensional Semiconductor Devices; Section VIII: Nonlinear Transport in Highly-Polar Semiconductors; Section IX: Nonlinear HigherOrder Thermo-Hydrodynamics, Section X: Coherent State and Nonequilibrium Bose-Einstein-Like Condensations; Section XI: Thermo-Statistics of Complex Structure Systems; Section XII: Non-Conventional Thermo-Hydrodynamics.

In conclusion the authors would like to acknowledge partial financial support received from the São Paulo State Research Agency (FAPESP), the Brazilian National Research Council (CNPq) [ARV, RL and CGR are CNPq Research Fellows] the Ministry of Planning-Finep, FAC-Unicamp, IBMBrasil, John Simon Guggleuheim Memorial Foundation (New York, USA). The author CGR thanks the financial support received from Goiás State Research Agency (FAPEG). AAPS and CABS are thankful to the DFMC-IFGW-Unicamp for the kind hospitality received during their stay there on a leave of absence provided by their Home Institutions.
[1] D.N. Zubarev, Nonequilibrium Statistical Thermodynamics (Plenum-Consultants Bureau, New York, USA, 1974); D.N. Zubarev Fortschr. Phys./Prog. Phys. 18, 125 (1970); D.N. Zubarev, Modern methods of the statistical theory of nonequilibrium processes, in Rewiews of Science and Technology: Moderns Problems of Mathematics, Vol. 15 (in Russian), pp. 131-226, edited by R.B. Gramkreludze (Izd. Nauka, Moscow, 1980) [English Transl. in J. Soviet Math. 16, 1509 (1981)].

[2] R. Zwanzig, Where we go from here?, in Perspectives in Statistical Mechanics, edited by H.J. Raveché (North Holland, Amsterdam, The Netherlands, 1981).

[3] R. Luzzi, A.R. Vasconcellos and J.G. Ramos, Predictive Statistical Mechanics: A Nonequilibrium Statistical Ensemble Formalism (Kluwer Academic, Dordrecht, The Netherlands, 2002).

[4] R. Luzzi, A.R. Vasconcellos and J.G. Ramos, The theory of irreversible processes: Foundations of a non-equilibrium statistical ensemble formalism, Rivista del Nuovo Cimento 29(2), 1-85 (2006).

[5] W. Heisenberg, Physics Today, May issue, pp. 22-23 (1967).

[6] R. Landauer, Nonlinearity: historical and technological view, in Nonlinearity in Condensed Matter, edited by A.R. Bishop, D.K. Campbell, P. Kumav and S.E. Trullinger (Springer, Berlin, Germany, 1987).

[7] G. Nicolis, Introduction to Nonlinear Science (Cambridge
Univ. Press, Cambridge, UK, 1995).

[8] H. Haken, Synergetics (Springer, Berlin, Germany, 1977).

[9] G. Nicolis and I. Prigogine, Self-Organization in Nonequilibrium Systems (Wiley-Interscience, New York, USA, 1977).

[10] G. Nicolis and I. Prigogine, Exploring Complexity (Freeman, New York, USA, 1989).

[11] S. Kauffman, The Origins of Order (Oxford Univ. Press, New York, USA, 1993).

[12] D. Jou, J. Casas-Vazquez and G. Lebon, Extended Irreversible Thermodynamics (Springer, Berlin, Germany, 1993; second edition, 1996, third enlarged edition, 2001); also Undenstanding Non-Equilibrium Thermodynamics (Springer, Berlin, Bermany, 2008).

[13] R. Luzzi, A.R. Vasconcellos and J.G. Ramos, Statistical Foundations of Irreversible Thermodynamics (TeubnerBertelsmann-Springer, Stuttgart, Germany, 2000).

[14] R. Luzzi, A.R. Vasconcellos and J.G. Ramos, Irreversible thermodynamics in a nonequilibrium statistical ensemble formalism, Rivista del Nuovo Cimento 24(3), 1-70 (2001).

[15] A.R. Vasconcellos, R. Luzzi and L.S. Garcia-Colin, Microscopic approach to irreversible thermodynamics. I. General theory, Phys. Rev. A 43, 6622 (1991);

A.R. Vasconcellos, R. Luzzi and L.S. Garcia-Colin, Microscopic approach to irreversible thermodynamics. II. An example from semiconductor physics, Phys. Rev. A 43, 6633 (1991); 
A.R. Vasconcellos, R. Luzzi and L.S. Garcia-Colin, Response function theory for thermal perturbations in informational statistical thermodynamics, Physica A 221, 478-494 (1995).

[16] M.A. Tenan, A.R. Vasconcellos and R. Luzzi, Fortschr. Phys./Prog. Phys. 47, 1-30 (1997).

[17] Articles in Nanotech: The science of the small gets down to business, Scientific American, Special issue, September (2001).

[18] G. Stix, Little big science: an overview (and articles thereafter), Scientific American 285, 26 (2001).

[19] J. Hopkings and W. Sibbet, Big payoffs in a flash, Scientific American 283, 54 (2000).

[20] R. Alfano, Frontiers of femtosecond and picosecond optical measuring techniques, in New Techniques and Ideas in Quantum Measurement, edited by D.M. Greenberger, Vol. 480 of NYAS Annals (New York Academy of Sciences, New York, USA, 1996).

[21] J.C. Diels and W. Rudolph, Ultrashort Laser Phenomena, Second updated and extended edition (Academic-Elsevier, Amsterdam, The Netherlands, 2006).

[22] C.H. Brito Cruz, Editor, special issue of Braz. J. Phys 26(2), June (1996).

[23] F. Family and T. Vizsek, Eds., Dynamic of Fractal Surfaces (World Scientific, Singapore, 1991).

[24] T.A. Witten, Insights from soft-condensed matter, in Rev. Mod. Phys. (Supplement Centenary APS) 71(2) 5367 (1999); T.A. Witten and T.A. Pincus, Structured Fluids (Oxford Univ. Press, Oxford, UK, 2004).

[25] O. Penrose, Foundations of statistical mechanics, Rep. Prog. Phys. 42(12), 1937 (1979).

[26] J.W. Gibbs, Elementary Principles in Statistical Mechanics (Yale Univ. Press, New Haven, USA, 1902; reprinted by Dover, New York, USA, 1960).

[27] R.P. Feynman, The Character of Physical Laws (MIT Press, Cambridge, USA, 1967).

[28] J. Bronowski, The Common Sense of Science (Harvard Univ. Press, Cambridge, USA, 1978).

[29] N. Oreskes, H. Shrader-Frechette and K. Beltz, Verification, Validation, and Confirmation of numerical models in the earth sciences, Science 263, 641-646 (1994); see also Refs. [98101].

[30] R. Kubo, Opening Address at the Oji Seminar, Prog. Theor. Phys. (Japan), Suppl. 64, 1-11 (1978).

[31] M.P. Allen and D.J. Tildesley, Computer Simulation of Liquids (Oxford Univ. Press, New York, USA, 1987).

[32] M.H. Kalos and P.A. Whitlock, Monte Carlo Methods (WileyInterscience, New York, USA, 2007);

K. Binder, Ed., Monte Carlo and Molecular Dynamic (Oxford univ. Press, Oxford, 1995);

P.B. Balbuena and J.M. Seminario, Eds. Molecular Dynamics (Elsevier, Amsterdam, The Netherlands, 1999);

D. Frenkel and B. Smit, Understanding Molecular Simulation (Academic, New York, USA, 2002).

[33] J. Langer, Computing in physics: are we taking it too seriously? Or not seriously enough?, Physics Today 52(7), pp. 11-13 (1999).

[34] B.J. Alder and T.E. Wainwright, Phase transition for a hard sphere system; studies in molecular dynamics. I. General method, J. Chem. Phys. 27, 1208 (1957); 31, 459 (1959).

[35] A. Rahman, Correlations in the motion of atoms in liquid argon, Phys. Rev. 136, issue 2A, pp. A405-A411 (1964).

[36] R. Car and M. Parrinello, Unified approach for molecular dynamics and density-functional theory, Phys. Rev. Lett. 55, 2471 (1985)

[37] W.G. Hoover, Molecular dynamics, in Lecture Notes in Physics, Vol. 258 (Springer, Berlin, Germany, 1986).
[38] J.G. Kirkwood, The statistical mechanical theory of transport processes I. General theory, J. Chem. Phys. 14, 180 (1946).

[39] M.S. Green, Markoff random processes and the statistical mechanics of time-dependent phenomena, J. Chem. Phys. 20, 1281 (1952).

[40] H. Mori, I. Oppenheim and J. Ross, Some topics in quantum statistics, in Studies in Statistical Mechanics I, edited by J. de Boer and G.E. Uhlenbeck (North Holland, Amsterdam, The Netherlands, 1962).

[41] H. Mori, Transport, collective motion, and Brownian motion, Prog. Theor. Phys. (Japan) 33(3), 423 (1965).

[42] R. Zwanzig, Statistical mechanics of irreversibility, in Lectures in Theoretical Physics, Vol. 3 (Wiley-Interscience, New York, USA, 1961).

[43] A. Salam, V.S. Vladimorov and A.A. Logunov, Tribute in Memoriam of Nicolai N. Bogoliubov, in Theor. Math. Phys. 92, 817 (1993).

[44] N.S. Krylov, Works on Foundations in Statistical Mechanics, with an Introduction by A.B. Migdal and V.A. Fock (Princeton Univ. Press, Princeton, USA, 1979).

[45] D.N. Zubarev, V. Morozov and G. Röpke, Statistical Mechanics of Nonequilibrium Processes, Vols. 1 and 2 (AkademieWiley VCH, Berlin, Germany, 1996 and 1997 respectively).

[46] S.V. Peletminskii and A.A Yatsenko, JETP 26, 773 (1968).

[47] A.I. Akhiezer and S.V. Peletminskii, Methods of Statistical Physics (Pergamon, Oxford, UK, 1981).

[48] R. Luzzi and A.R. Vasconcellos, Fortschr. Phys./Prog. Phys. 38, 887 (1990).

[49] R. Luzzi, A.R. Vasconcellos and J.G. Ramos, A nonequilibrium statistical ensemble formalism MaxEnt-NESOM: basic concepts, construction, application, open questions and criticisms, Int. J. Mod. Phys. B 14(28), 3189 (2000).

[50] D.N. Zubarev and V.P. Kalashnikov, Theor. Math. Phys. 1, 108 (1971).

[51] C.E. Shannon, A mathematical theory of communication, Bell System Technical Journal 27, July issue, 379-423 (1948), and October issue, 623-656 (1948);

also in Claude Elwood Shannon: Collected Papers, edited by N.J.A. Sloane and A.D. Wyner (Wiley-Interscience, New York, USA, 1993).

[52] C.E. Shannon and W. Weaver, The Mathematical Theory of Communication (University Illinois Press, Urbana, USA, 1949).

[53] L. Brillouin, Science and Information Theory (Academic, New York, USA, 1962).

[54] N.N. Bogoliubov, Problems of a dynamical theory in statistical physics, in Studies in Statistical Physics, edited by J. de Boer and G.E. Uhlenbeck (North Holland, Amsterdam, The Netherlands, 1962).

[55] R. Peierls, Some simple remarks on the basis of transport theory, in Transport Phenomena, Lecture Notes in Physics, Vol. 31, edited by J. Ehlers, K. Hepp and H.A. Weidenmuller (Springer, Berlin, Germany, 1974)

[56] U. Fano, Description of states in quantum mechanics by density matrix and operator techniques, Rev. Mod. Phys. 29, 74 (1957).

[57] B. Robertson, Introduction to field operators in quantum mechanics, Am. J. Phys. 41, 678 (1973).

[58] N.N. Bogoliubov, Lectures in Quantum Mechanics, Vols. 1 and 2 (Gordon and Breach, New York, USA, 1967 and 1970 respectively).

[59] L. Lauck, A.R. Vasconcellos and R. Luzzi, A nonlinear quantum transport theory, Physica A 168, 789 (1990).

[60] W.M. Elsasser, On quantum measurements and the role of the uncertainty relations in statistical mechanics, Phys. Rev. 52, 987 (1937). 
[61] E.T. Jaynes, in Physics and Probability, edited by W.T. Grandy and P.W. Milmmi (Cambridge Univ. Press, Cambridge, UK, 1993), pp. 261-275.

[62] H. Haken, in ibid. Ref. [70], pp. 239-250.

[63] B. Robertson, in ibid. Ref. [70], pp. 251-260.

[64] E.T. Jaynes, Probability Theory as Extended Logic (Cambridge Univ. Press, Cambridge, UK, 2002).

[65] J. Bricmont, in Chance in Physics, edited by J. Bricmont et al., (Springer, Heidelberg, Germany, 2001);

J. Bricmont, Physicalia Magazine 17, 159 (1995) and in The Flight from Science and Reason, Annals New York Academy of Sciences, Vol. 775, pp. 131-175 (NYAS, New York, USA, 1996).

[66] R. Courant and D. Hilbert, Methods of Mathematical Physics (Wiley-Interscience, New York, USA, 1953).

[67] J.R. Madureira, A.R. Vasconcellos, R. Luzzi and L. Lauck, Markovian kinetic equations in a nonequilibrium statistical ensemble formalism, Phys. Rev. E 57, 3637 (1998).

[68] J.R. Madureira, A.R. Vasconcellos, R. Luzzi, J. CasasVazquez and D. Jou, Evolution of dissipative processes via a statistical thermodynamic approach. I. Generalized MoriHeisenberg-Langevin equations, J. Chem. Phys. 108, 7568 (1998).

[69] J.G. Ramos, A.R. Vasconcellos and R. Luzzi, Fortschr. Phys./Prog. Phys. 43, 265 (1995).

[70] E.B. Davies, Commun. Math. Phys. 39, 91 (1994).

[71] C.A.B. Silva, J.G. Ramos, A.R. Vasconcellos and R. Luzzi, Kinetic Equations for Far-From-Equilibrium Many-Body Systems, IFGW-Unicamp Internal Report (2008) and Europhys. Lett., submitted.

[72] H. Spohn, Rev. Mod. Phys. 53, 569 (1980).

[73] Yu. L. Klimontovich, Statistical Theory of Open Systems, Volume 1: A Unified Approach to Kinetic Description of Processes in Active Systems (Kluwer Academic, Dordrecht, The Netherlands, 1995).

[74] H.J. Kreuzer, Nonequilibrium Thermodynamics and its Statistical Foundations (Claredon, Oxford, UK, 1981).

[75] H. Fröhlich, Phys. Lett. A 51, 21-22 (1975); M.V. Mesquita, A.R. Vasconcellos and R. Luzzi, Amplification of coherent polar vibrations in biopolymers: Fröhlich condensate, Phys. Rev. E 48, 4049-4059 (1993).

[76] D. Snoke, Condensed-matter physics: coherent questions, Nature 443, 403-404 (2006).

[77] C.A.B. Silva, R.M.O. Galvão, Phys. Rev. E 60, 7435-7440 (1999).

[78] R. Luzzi and A.R. Vasconcellos, Ultrafast transient response of nonequilibrium plasma in semiconductors, in Semiconductor Processes Probed by Ultrafast Laser Spectroscopy, Vol. 1, edited by R.R. Alfano, (Academic, New York, 1984), pp. 135-169;

C.G. Rodrigues, A.R. Vasconcellos and R. Luzzi, A kinetic theory for nonlinear tuantum transport, Transp. Theor. Stat. Phys. 29, 733 (2000).

[79] O.S. Duarte and A.O. Caldeira, Effective coupling between two Brownian particles, Phys. Rev. Lett. 97, 250601 (2006).

[80] R. Balescu, Equilibrium and Nonequilibrium Statistical Mechanics (Wiley-Interscience, New York, USA, 1975).

[81] F. Reif, Foundations of Statistical and Thermal Physics (McGraw-Hill, New York, USA, 1965).

[82] J.C. Maxwell, Phil. Trans. Roy. Soc. (London) 157, 49 et seq.

[83] H. Grad, Comm. Pure Appl. Math. 2, 331 (1949); ibid. 5, 455 (1952).

[84] H. Grad, Principles of the Kinetic Theory of Gases, in Handbuch der Physik Vol. XII, S. Flügge, Ed., pp. 205-294 (Springer, Berlin, Germany, 1958).

[85] C.A.B. Silva, J.G. Ramos, A.R. Vasconcellos and R. Luzzi.
Nonlinear Higher-Order Hydrodynamics: Unification of Kinetic and Hydrodynamic Approaches within a Nonequilibrium Statistical Ensemble Formalism, IFGW-Unicamp Internal Report (2007) and future publications.

[86] S.J. Gould, Dinosaur in a Haystack (Random House, New York, 1995).

[87] M. Born, Experiment and Theory in Physics (Dover, New York, 1956).

[88] J.T. Cushing, Philosophical Concepts in Physics (Cambridge Univ. Press., Cambridge, 1998).

[89] W. Heisenberg, The Physical Conception of Nature, (Hutchinson, London, 1958).

[90] W. Heisenberg, The notion of a "close theory" in modern science, in Across the Frontiers, edited by Ruth N. Anshen (Harper and Row, New York, 1975), pp. 184-191.

[91] S. Hawking, The Nature of Space and Time (Princeton Univ. Press, Princeton, 1996).

[92] R. Luzzi, A.R. Vasconcellos and J.G. Ramos, A NonEquilibrium Statistical Ensemble Formalism: Basic Concepts, Construction, Applications, Open Questions and Criticism, Int. J. Mod. Phys. B 14(3), 189-264 (2000); also http://xxx.lanl.gov/abs/cond-mat/9909160.

[93] R.R. Alfano, editor, Biological Events Probed by Ultrafast Laser Spectroscopy (Academic Press, New York, 1982).

[94] R.R. Alfano, editor, Semiconductors Probed by Ultrafast Laser Spectroscopy, Vols. 1 and 2 (Academic Press, New York, 1984 and 1985 respectively).

[95] J.S. Driels and W. Rudolph, Ultrafast laser pulse phenomena, in the series Optics and Photonic (Academic, New York, 1996).

[96] C.B. Harris, E.P. Ippen, G.A. Mourou and A.H. Zewwail, Ultrafast Phenomena (Springer, Berlin, 1989).

[97] J.M. Hopkings and W. Sibbet, Ultrashort-pulse lasers: big payoffs in a flash, Scientific American 283(3), 54-61 (2000).

[98] C.V Shank, Measurement of ultrafast phenomena in the femtosecond time domain, Science 219, pp. 1027-1031 (1983).

[99] R. Luzzi, Ultrafast relaxation processes in semiconductors, in High Excitation and Short Pulse Phenomena, edited by M. Pilkhun (North Holland, Amsterdam, 1985).

[100] R. Luzzi, Nonequilibrium plasma in photoexcited semiconductors, in Recent Developments in Nonequilibrium Thermodynamics, edited by J. Casas-Vazquez and D. Jou (Springer, Berlin, 1986); A.C. Algarte, A.R. Vasconcellos and R. Luzzi, Braz. J. Phys. 26, 543 (1996).

[101] T. Hill, Thermodynamic of small systems, J. Chem. Phys. 36, 3182 (1962).

[102] M.C. Roco, Nanotechnology's Future, in Forum Section of Scientific American 295(2), p. 21, August (2006).

[103] N.M. Johnson, A.V. Nurmikko, and S.P. DenBaars, Blue diode lasers, Physics Today 53 (10), pp. 31-36 (2000).

[104] G. Zorpette, Blue chips, in the Profile Section of Scientific American 283 (2), pp. 19-20 (2000).

[105] S.J. Pearson (ed.), GaN and Related Materials (Optoelectronic Properties of Semiconductors and Superlattices) (New York: Gordon and Breach, 1997).

[106] S. Nakamura and G. Fasol, The Blue Laser Diode (Springer, Berlin, 1997).

[107] S.N. Mohammad and H. Morkoç, Progress and prospects of group-III nitride semiconductors, Prog. Quantum Electron. 20(5-6), 361 (1996).

[108] I. Akasaki and H. Amano, Crystal growth and conductivity control of group III nitride semiconductors and their application to short wavelength light emitters, Japan. J. Appl. Phys. 36, 5393-5408 (1997).

[109] K. Moore and R.J. Trew, Radio-frequency power transistors based on $6 H$ - and $4 H-S i C$, Mater. Res. Soc. Bull. 22(3), 50 
(1997).

[110] M.A. Khan, J.N. Kuznia, D.T. Olson, W.J. Schaff, J.W. Burm and M.S. Shur, Microwave performance of a $0.25 \mu \mathrm{m}$ gate AlGaN/GaN heterostructure field effect transistor, Appl. Phys. Lett. 65, 1121 (1994).

[111] M.A. Khan, Q. Chen, J.W. Yang, M.S. Shur, B.T. Dermott and J.A. Higgins, IEEE Electron. Device Lett. 17, 325 (1996).

[112] M.S. Shur and M.A. Khan, $\mathrm{MoSe}_{3} S$ : a novel insertion cathode for secondary lithium batteries, Mater. Res. Bull. 22(1), 44 (1997).

[113] Q. Chen, J.W. Yang, R. Gaska, M.A. Khan, M.S. Shur, G.J. Sullivan, A.L. Sailor, J.A. Higgins, Ping A.T. and I. Adesida, IEEE Electron. Device Lett. 19, 44 (1998).

[114] K. Shenai, R.S. Scott and B.J. Baliga IEEE Trans. Electron. Devices 36, 1811 (1989).

[115] H. Morkoç, R. Cingolani, W. Lambrecht, B. Gil, H-X Jiang, J. Lin, D. Pavlidis and K. Shenai, MRS Internet J. Nitride Semicond. Res. 4, U23-31 (1999).

[116] D.K. Ferry, High-field transport in wide-band-gap semiconductors, Phys. Rev. B 12, 2361 (1974);

M.A. Littlejohn, J.R. Hauser and T.H. Glisson, Monte Carlo calculation of the velocity-field relationship for gallium nitride, Appl. Phys. Lett. 26, 625 (1975);

J. Kolník , I.H. Oğuzman, K.F. Brennan, R. Wang, P.P. Ruden and Y. Wang, Electronic transport studies of bulk zincblende and wurtzite phases of GaN based on an ensemble Monte Carlo calculation including a full zone band structure, J. Appl. Phys. 78, 1033 (1995);

M. Shur, B. Gelmont and M.A. Khan, J. Electron. Mater. 25 , 777 ( 1996);

S.K. O'Leary, B.E. Foutz, M.S. Shur, U.V. Bhapkar and L.F. Eastman, Electron transport in wurtzite indium nitride, J. Appl. Phys. 83, 826 (1998);

E. Bellotti, B.K. Doshi, K. Brennan, J.D. Albrecht and P.P. Ruden, Ensemble Monte Carlo study of electron transport in wurtzite InN, J. Appl. Phys. 85, 916 (1999).

[117] B.K. Ridley, B.E. Foutz and L.F. Eastman, Phys. Rev. B 61 16862 (2000);

J.W. Orton and C.T. Foxon, Semicond. Sci. Technol. 13, 310 (1998);

V.W. Chin, T.L. Tansley and T. Osotchan, Electron mobilities in gallium, indium, and aluminum nitrides, J. Appl. Phys. 75, 7365 (1994);

S. Dhar and S. Ghosh, Low field electron mobility in GaN, J. Appl. Phys. 86, 2668 (1999);

D.C. Look and R.J. Molnar, Appl. Phys. Lett. 70, 3377 (1997).

[118] M. Wraback, H. Shen, J.C. Carrano, T. Li, J.C. Campbell, M.J. Schurman and I.T. Ferguson, Time-resolved electroabsorption measurement of the electron velocity-field characteristic in GaN, Appl. Phys. Lett. 76, 1155 (2000);

M. Wraback, H. Shen, J.C. Carrano, C.J. Collins, J.C. Campbell, R.D. Dupuis, M.J. Schunnan and I.T. Ferguson, Timeresolved electroabsorption measurement of the transient electron velocity overshoot in GaN, Appl. Phys. Lett. 79, 1303 (2001);

R. Collazo, R. Schlesser, A. Roskowski, R.F. Davis and Z. Sitar, Hot electron transport in AlN, J. Appl. Phys. 88, 5865 (2000);

R. Collazo, R. Schlesser and Z. Sitar, Experimental observation of electron velocity overshoot in AlN, Appl. Phys. Lett. 81, 5189 (2002).

[119] N. Mansour, K.W. Kim, N.A. Bannov and M.A. Littlejohn, Transient ballistic transport in GaN, J. Appl. Phys. 81, 2901 (1997).

[120] B.E. Foutz, L.F. Eastman, U.V. Bhapkar and M.S. Shur, Com- parison of high field electron transport in GaN and GaAs, Appl. Phys. Lett. 70, 2849 (1997).

[121] E.W.S. Caetano, R.N.C. Filho, V.N. Freire and J.A.P. Costa, Velocity overshoot in zincblende and wurtzite GaN, Solid State Commun. 11099), 469 (1999).

[122] B.E. Foutz, S.K. O'Leary, M.S. Shur and L.F. Eastman, Transient electron transport in wurtzite GaN, InN, and AlN, J. Appl. Phys. 85, 7727 (1999).

[123] C.G. Rodrigues, V.N. Freire, A.R. Vasconcellos and R. Luzzi, Velocity overshoot onset in nitride semiconductors, Appl. Phys. Lett. 76, 1893 (2000).

[124] C.G. Rodrigues, A.R. Vasconcellos, R. Luzzi and V.N. Freire, Transient transport in III-nitrides: interplay of momentum and energy relaxation times, J. Phys.: Condens. Matter 19, 346214 (2007).

[125] C.G. Rodrigues, V.N. Freire, J.A.P. Costa, A.R. Vasconcellos, R. Luzzi, Hot electron dynamics in zincblende and wurtzite GaN, Phys. Stat. Sol. (b) 216, 35 (1999);

C.G. Rodrigues, A.R. Vasconcellos, R. Luzzi, V. Lemos, V.N. Freire, Structured ultrafast carrier drift velocity in photoexcited zincblende GaN, Materials Science Forum 338-342, 1579 (2000);

C.G. Rodrigues, A.R. Vasconcellos, V.N. Freire, R. Luzzi, $U r$ bach's tail in III-nitrides under an electric field, J. Appl. Phys. 90, 1879 (2001);

C.G. Rodrigues, V.N. Freire, A.R. Vasconcellos, R. Luzzi, Electron mobility in nitride materials, Braz. J. Phys. 32(2A), 439 (2002)

A.R. Vasconcellos, R. Luzzi, C.G. Rodrigues, V.N. Freire, Hot phonon bottleneck in the photoinjected plasma in GaN, Appl. Phys. Lett. 82, 2455 (2003);

C.G. Rodrigues, J.R.L. Fernandez, J.R. Leite, V.A. Chitta, V.N. Freire, A.R. Vasconcellos, R. Luzzi, Hole mobility in zincblende GaN, J. Appl. Phys. 95, 4914 (2004);

C.G. Rodrigues, A.R. Vasconcellos, R. Luzzi, Ultrafast relaxation kinetics of photoinjected plasma in III-nitrides, J. Phys. D - Appl. Phys. 38, 3584 (2005);

C.G. Rodrigues, A.R. Vasconcellos, R. Luzzi, V.N. Freire, Nonlinear transport properties of III-nitrides in electric field, J. Appl. Phys. 98, 043702 (2005);

C.G. Rodrigues, A.R. Vasconcellos, R. Luzzi and V.N. Freire, Nonlinear transport properties of doped III-N and GaAs polar semiconductors: a comparison, J. Appl. Phys. 98, 043703 (2005);

C.G. Rodrigues, A.R. Vasconcellos, R. Luzzi, Nonlinear charge transport in III-N semiconductors: mobility, diffusion, and a generalized Einstein relation, J. Appl. Phys. 99, 73701 (2006);

C.G. Rodrigues, A.R. Vasconcellos, R. Luzzi, Non-linear electron mobility in n-doped III-nitrides, Braz. J. Phys. 36(2A), 255 (2006)

C.G. Rodrigues, A.R. Vasconcellos, R. Luzzi, Nonlinear transport in n-III-nitrides: selective amplification and emission of coherent LO phonons, Solid State Commun. 140, 135 (2006);

C.G. Rodrigues, Electron drift velocity in $n$-doped wurtzite GaN, Chinese J. Phys. 44, 44 (2006);

C.G. Rodrigues, Electron mobility in n-doped zinc sulphide, Microelectronics Journal 37, 657 (2006);

C.G. Rodrigues, A.R. Vasconcellos, R. Luzzi, Nonlinear hole transport and nonequilibrium thermodynamics in group IIInitrides under the influence of electric fields, J. Appl. Phys. 102, 073714 (2007);

C.G. Rodrigues, Electron transport in $G a N(Z B)$ and AlN(WZ), J. Mater. Sci. 42, 396 (2007);

C.G. Rodrigues, Hot-carrier relaxation in photoinjected 
ZnSe, Microelectronics Journal 38, 24 (2007);

C.G. Rodrigues, Ultrafast transport transient in photoexcited ZnSe, Eur. Phys. J. Appl. Phys. 41, 201 (2008);

C.G. Rodrigues, A.R. Vasconcellos, R. Luzzi, Theoretical calculations of nonlinear electronic transport behavior in IIInitrides: GaN and AlN, Phys. Status Solidi (b) 246(2), 417 (2009);

C. G. Rodrigues, A. R. Vasconcellos, R. Luzzi, Optical properties of III-nitrides in electric fields, Eur. Phys. J. B 72, 67 (2009);

C.G. Rodrigues, Influence of the concentration, temperature and electric field intensity on the electron mobility in $n$-doped zinc sulphide, Eur. Phys. J. B 72(3), 405 (2009);

[126] R. Wallace, The World of Leonardo (Time-Life, New York, USA, 1966).

[127] J.P. Boon and S. Yip, Molecular Hydrodynamics (McGrawHill, New York, USA, 1980; Reprinted by Dover, New York, USA, 1991).

[128] S.K. Belyaev, Phys.-Uspekhi 38, 287 (1995).

[129] D.N. Zubarev, V.G. Morosov, I.P. Omelyan, and M.V. Tokarchuk, Theor. Math. Phys. 96, 997 (1994).

[130] H.G.B. Casimir, On Onsager's principle of microscopic reversibility, Rev. Mod. Phys. 17, 343 (1945).

[131] I. Müller and T. Ruggeri, Extended Thermodynamics (Springer, Berlin, Germany, 1993).

[132] D. Jou, J.Casas-Vazquez, and G. Lebon, Rep. Prog. Phys. 51, 1105 (1998) and ibid. 62, 1035 (1999).

[133] D. Jou, J. Casas-Vazquez, J.R. Madureira, A.R. Vasconcellos, and R. Luzzi, Higher-order hydrodynamics: Extended Fick's Law, evolution equation, and Bobylev's instability, J. Chem. Phys. 116(4), 1571 (2002).

[134] D. Jou, J. Casas-Vazquez, J.R. Madureira, A.R. Vasconcellos, and R. Luzzi, J. Mod. Phys. B 32, 4211 (2001).

[135] S. Hess, Z. Naturforsh. A 32, 678 (1977).

[136] C. Wunsch, Oceanography: what is the thermohaline circulation?, Science 298, 1179-1181 (2002).

[137] J.D. Neelin and M. Latif, El Niño dynamics, Phys. Today 51(12), pp. 32-36 (1998).

[138] C-L Chiu, J. Hydraulic. Eng. 129, 248 (2003).

[139] D. Jou, J. Casas-Vazquez and Criado-Sancho, Thermodynamics of Fluids Under Flow (Springer, Berlin, Germany, 2001).

[140] V. Garzó and A. Santos, Kinetic Theory of Gases in Shear Flow (Kluwer Academic, Dordrecht, The Netherlands,2003).

[141] A.R. Vasconcellos, A.A.P. Silva, R. Luzzi, J.G. Ramos, D. Jou and J. Casas-Vazquez, Hydrodynamic Motion in ComplexStructured Materials, IFGW-Unicamp Internal Report (2008); and future publication (see also Ref. [266]).

[142] A.M. Anile and O. Muscato, Phys. Rev. B 51, 17628 (1995).

[143] F.J. Uribe, R.M. Velazco, L.S. Garcia-Colin, and E.D. Herrera, Shock wave profiles in the Burnett approximation, Phys. Rev. E 62, 6648 (2000).

[144] F.J. Uribe and L.S. Garcia-Colin, Burnett description for plane Poiseuille flow, Phys. Rev E 60, 4063 (1999), and references therein.

[145] D.W. Mackowski, D.H. Papadopolous, and D.E. Roner, Phys. Fluids 11, 2108 (1999), and references therein.

[146] A.V. Bobylev, Doklady 27, 29 (1982).

[147] F.J. Uribe, R.M. Velasco, and L.S. Garcia-Colin, Bobylev's instability, Phys. Rev. E 62, 5835 (2000).

[148] L.V. Karlin, J. Phys. A: Mat. Gen. 33, 8037 (2000).

[149] J. Eggers, Hydrodynamic Singularities, in A Perspective Look at Nonlinear Media, J. Parisi, S.C. Múller, and W. Zimmerman, Editors (Springer, Berlin, Germany, 1998).

[150] C. Godreche and P. Manneville, Hydrodynamic and Nonlinear Instabilities (Cambridge Univ. Press, New York, USA, 1998).

[151] L. Sklar, Physics and Chance: Philosophical Issues in the
Foundations of Statistical Mechanics (Cambridge Univ. Press, Cambridge, UK, 1993).

[152] J.P. Dougherty, Phil. Trans. Roy. Soc. (London) A 346, 259 (1994).

[153] P. Glandsdorff and I. Prigogine, Thermodynamics Theory of Structure, Stability and Fluctuations (Wiley-Interscience, London, UK, 1971).

[154] S. Hawling and D. Ben Avraham, Adv. Phys. 36, 695 (1987).

[155] J.R. Dutcher and A.G. Marangoni, Soft Materials (Marcel Dekker, New York, USA, 2005).

[156] J. Crank, The Mathematics of Diffusion (Oxford Univ. Press, Oxford, UK, 1975).

[157] I. Prigogine and I. Stengers, Order Out of Chaos (Bantam, New York, USA, 1984).

[158] M.V. Mesquita, A.R. Vasconcellos and R. Luzzi, Amplification of coherent polar vibrations in biopolymers: Fröhlich condensate, Phys. Rev. E 48, 4049 (1993).

[159] M.V. Mesquita, A.R. Vasconcellos and R. Luzzi, Phys. Rev. E 58, 7993 (1998).

[160] A.R. Vasconcellos, M.V. Mesquita and R. Luzzi, Europhys. Lett. 49, 637 (2000).

[161] A.J. Kent et al., Acoustic phonon emission: observation of phonon resonance, Phys. Rev. Lett. 96, 215504 (2006).

[162] C.G. Rodrigues, A.R. Vasconcellos and R. Luzzi, Emission of coherent A-phonons generated by drifting electrons: "SASER", future publication.

[163] C.G. Rodrigues, A.R. Vasconcellos and R. Luzzi, Nonequilibrium Fröhlich-Bose-Einstein Condensation and Laser-like Behavior of LO-Phonons Generated by Drifting Electrons, IFGW-Unicamp Internal Report (2009) and future publications.

[164] A.R. Vasconcellos, R. Luzzi and J.R. Madureira, Optical properties of semiconductors via pump-probe experiments: statistical-thermodynamical approach, hot plasma and coherent phonon states, J. Phys. Cond. Matt. 12(24), 5325 (2000).

[165] G. Nicolis, Physics of far-from-equilibrium systems and selforganization, in The New Physics, editor P. Davies, pp. 316347 (Cambridge Univ. Press, Cambridge, 1989).

[166] I. Prigogine and I. Stengers, Order out of the Chaos: Man's New Dialogue with Nature (Bantam, New York, 1984).

[167] I. Prigogine, Dissipative structures, dynamics, and entropy, Int. J. Quantum Chem. 9, 443-456 (1975).

[168] A.F. Fonseca, M.V. Mesquita, A.R. Vasconcellos and R. Luzzi, Informational-statistical thermodynamics of a complex system, J. Chem. Phys. 112, 3967-3979 (2000).

[169] M.V. Mesquita, A.R. Vasconcellos, R. Luzzi and S. Mascarenhas, Systems Biophysics, Braz. J. Phys. 34, 459 (2004).

[170] A.R. Vasconcellos, M.V. Mesquita and R. Luzzi, Complex behavior in biosystems: an information-theoretic approach, Chaos, Solitons, Fract. 11, 1313-1325 (2000).

[171] M.V. Mesquita, A.R. Vasconcellos and R. Luzzi, Considerations on Fröhlich's nonequilibrium Bose-Einstein-like condensation, Phys. Lett. A 238, 206-211 (1998).

[172] M.V. Mesquita, A.R. Vasconcellos and R. Luzzi, Positivefeedback-enhanced Fröhlich's condensation in biosystems, Int. J. Quantum Chem. 66, 177-187 (1998).

[173] L. Lauck, A.R. Vasconcellos and R. Luzzi, On Fröhlich's coherent effects in biological systems: influence of carriers and high-order dissipative effects, J. Theor. Biol. 158, 1-13 (1992).

[174] H. Fröhlich, Evidence for Bose-condensation-like excitation of coherent modes in biological systems, Phys. Lett. A 51, 2122 (1975).

[175] A.C. Scott, F.Y. Chu and S.W. McLaughlin, The soliton: a new concept in applied science, Proc. IEEE 61, 1443-1483 (1973). 
[176] A.J. Heger, S. Kivelson, J.R. Schrieffer and W.P. Su, Solitons in conducting polymers, Rev. Mod. Phys. 60, 781-850 (1988).

[177] D.R. Herschbach, The next 35 years of chemistry, The Sciences, Suppl. Fin-de-siècle perspectives, December (1996).

[178] A.S. Kivelson, Soliton model of polyacetylene, in Solitons, editors S. E. Trullinger, V. E. Zahkarov and V. L. Prokovsky, pp. 301-387 (Elsevier, New York, 1986).

[179] K. Leutwyler, Plastic power, Scientific American, pp. 25-27, December (1996).

[180] A.S. Davydov, Theory of contraction of proteins under their excitation, J. Theor. Biol. 38, 559-69 (1973).

[181] A.R. Vasconcellos and R. Luzzi, Vanishing thermal damping of Davydov's solitons, Phys. Rev. E 48, 2246-2249 (1993).

[182] M.V. Mesquita, A.R. Vasconcellos and R. Luzzi, Neardissipationless coherent excitations in biosystems, Int. J. Quantum Chem 60, 689-697 (1996).

[183] A.R. Vasconcellos, M.V. Mesquita and R. Luzzi, Solitary wave excitation in acetanilide, Phys. Rev. Lett. 80, 2008-2011 (1998).

[184] A.R. Vasconcellos and R. Luzzi, Complex behavior in condensed matter: morphological ordering in dissipative carrier systems, Complexity 2(5), 42-49 (1997).

[185] S.A. Hassan, A.R. Vasconcellos and R. Luzzi, Nonlineardriven instability of dynamical plasma in solids: emergence of spatially self-organized order and chaotic-like behavior, Eur. Phys. J. B 13, 131-139 (2000).

[186] E.W. Montroll and M.F. Shlesinger, J. Stat. Phys. 12, 209 (1983).

[187] R.A. Fisher, Phil. Trans. Roy. Soc. London A 222, 309 (1922).

[188] R. Balian, Incomplete descriptions and relevant entropies, Am. J. Phys. 67(12), 1078-1090 (1999).

[189] P.T. Landsberg, Entropies Galore!, Braz. J. Phys. 29(1), 46 (1999).

[190] G.M. Zaslavsky, Chaos, fractional kinetics, and anomalous transport, Phys. Rep. 371(6), pp. 461-580 (2002).

[191] M.F. Shlesinger, G.M. Zaslavsky and U. Frish, Lèvy, Flights and Related Topics in Physics (Springer, Berlin, Germany, 1985).

[192] W. Ebeling, Statistical Physics and Thermodynamics of Nonlinear Non-equilibrium Systems (World Scientific, Singapore, 1993).

[193] R. Feistel and W. Ebeling, Evolution of Complex Systems (Kluwer Academic, Dordrecht, The Netherlands, 1989).

[194] W. Ebeling, On the relation between various entropy concepts and the valoric interpretation, Physica A 182, 108-120 (1992).

[195] C. Beck and E.G.D. Cohen, arXiv.org/cond-mat/0205097 (2002).

[196] J. Havrda and F. Charvat, Kybernetica 3, 30 (1967).

[197] S. Abe and Y. Okamoto, in Nonextensive Statistical Mechanics and its Applications, edited by S. Abe and Y. Okamoto (Springer, Berlin, Germany, 2001).

[198] A. Renyi, "On Measures of entropy and Information", Proc. 4th Berkeley Symp. Math. Stat. Problems 1, 547 (1961).

[199] F. Takens and E. Verbitski, Generalized entropies: Rényi and correlation integral approach, Nonlinearity 11(4), 771 (1998).

[200] P. Grassberger and I. Procaccia, Estimation of the Kolmogorov entropy from a chaotic signal, Phys. Rev. A 28, 2591 (1983).

[201] P. Grassberger, Information flow and maximum entropy measures for 1-D maps, Physica D 14(3), 365-373 (1985).

[202] P. Jizba and T. Arimitzu, Ann. Phys. 312, 17 (2004) and also arXiv.org/cond- mat/0207707 and 0307698.

[203] B.D. Sharma and D.P. Mittal, J. Math. Sci. 10, 28 (1975).

[204] V.M. Vasyliunas, J. Geophys. Res. 73, 2839 (1968).

[205] G. Kaniadakis, Phys. Rev. E 66, 56125 (2002).
[206] E.T. Jaynes, in E. T. Jaynes Papers on Probability, Statistics, and Statistical Physics, edited by R. D. Rosenkrantz (ReidelKluwer Academic, Dordrecht, The Netherlands, 1983).

[207] E.T. Jaynes, in Frontiers of Non-equilibrium Statistical Physics, edited by G.T. Moore and M.O. Scully (Plenum, New York, USA, 1986), pp. 33-55.

[208] R.T. Cox, The Algebra of Probable Inference (John Hopkings Press, Baltimore, USA, 1961). 109

[209] E.T. Jaynes, Probability Theory: The Logic of Science (Cambridge Univ. Press, Cambridge, UK, 2002).

[210] S. Kulback and R.A. Leibler, Ann. Math. Stat. 22, 79 (1951).

[211] R. Balian and N.L. Balazs, Ann. Phys. 179, 97 (1987).

[212] W.T. Grandy, Principles of Statistical Mechanics: Equilibrium Theory (Reidel-Kluwer Academic, Dordrecht, The Netherlands, 1987).

[213] W.T. Grandy, Principles of Statistical Mechanics: Nonequilibrium Phenomena (Reidel-Kluwer Academic, Dordrecht, The Netherlands, 1988).

[214] W. Ochs, Rep. Math. Phys. 8, 109 (1975).

[215] P.T. Landsberg and V. Vedral, Distributions and channel capacities in generalized statistical mechanics, Phys. Lett. A 247, 211-217 (1998).

[216] J.N. Kapur and H.K. Kesavan, Entropy Optimization Principles with Applications (Academic, Boston, USA, 1992).

[217] P.S. Laplace, Essay Philosophique sur les Probabilities (Bachelier, Paris, France, 1825): English translation: A Philosophical Essay on Probability (Reprint by Dover, New York, USA, 1995).

[218] E.T. Jaynes, in The Maximum Entropy Formalism, edited by M. Tribus and R. D. Levine (MIT Press, Cambridge, MA, USA, 1978), pp. 15-118.

[219] L. Onsager, Reciprocal relations in irreversible processes I, Phys. Rev. 37, 405 (1931).

[220] E.T. Jaynes, Information theory and statistical mechanics, Phys. Rev. 106, 620 (1957).

[221] E.T. Jaynes, Information theory and statistical mechanics. II, Phys. Rev. 108, 171 (1957).

[222] E.T. Jaynes, in Maximum Entropy and Bayesian Methods, edited by J. Skilling (Kluwer Academic, Dordrecht, The Netherlands, 1989), pp. 1-27.

[223] E.T. Jaynes, in Maximum Entropy and Bayesian Methods, edited by W. T. Grandy and L. H. Schick (Kluwer Academic, Dordrecht, The Netherlands, 1991), pp. 1-13.

[224] H. Jeffreys, Probability Theory (Clarendon, Oxford, UK, 1961).

[225] H. Jeffreys, Scientific Inference (Cambridge Univ. Press, Cambridge, UK, 1973).

[226] J. Uffink, Stud. Hist. Phil. Mod. Phys. 26, 223 (1995).

[227] R. Landauer, Inadequacy of entropy and entropy derivatives in characterizing the steady state, Phys. Rev. A 12, 636 (1975).

[228] M. Bunge, In praise of intolerance to charlatanism in academia, in The Flight From Science and Reason, eds. P. R. Gross, N. Levit and M. Lewis, Annals of the New York Academy of Science, Vol. 665, p. 112 (NYAS, New York, USA, 1996)

[229] J. Bricmont, Science of Chaos or Chaos in Science?, in The Flight From Science and Reason, eds. P. R. Gross, N. Levit and M. Lewis, Annals of the New York Academy of Science, Vol. 665 (NYAS, New York, USA, 1996).

[230] P.W. Anderson, The reverend Thomas Bayes, needles in haystacks, and the fifth force, Phys. Today 45(1), pp. 9-11 (1992).

[231] S. Hawkings, 1990 - Yearbook of Science and Future (Encyclopaedia Britannica, Chicago, USA, 1989).

[232] L. Sklar, Theory and Truth: Philosophical Critique Within 
Foundational Science (Oxford Univ. Press, Oxford, UK, 2000).

[233] B.C. Fraassen, British J. Phil. Sc. 32, 375 (1981).

[234] B.C. Fraassen, R.I. Hughes, and G. Herman, British J. Phil. Sc. 37, 453 (1986).

[235] B.C. Fraassen, Laws and Symmetry (Claredon, Oxford, UK, 1989).

[236] J.D. Herniter, Jour. Market Res. 10, 361 (1973).

[237] A. Hobson, J. Chem. Phys. 45, 1352 (1966).

[238] A. Hobson, Am. J. Phys. 34, 411 (1966).

[239] L.S. Garcia-Colin, A.R. Vasconcellos, and R. Luzzi, J. NonEquilib. Thermodyn. 19, 24 (1994).

[240] B. Robertson, Equations of motion in nonequilibrium statistical mechanics, Phys. Rev. 144, 151 (1966);

B. Robertson, Equations of motion in nonequilibrium statistical mechanics. II. energy transport 160, 175 (1967).

[241] R. Luzzi and A.R. Vasconcellos, Response function theory for far-from-equilibrium statistical systems, J. Stat. Phys. 23(5), 539-559 (1980).

[242] E.T. Jaynes, Am. J. Phys. 33, 391 (1965).

[243] E.P. Gyftopoulos and E. Çubukçu, Entropy: thermodynamic definition and quantum expression, Phys. Rev. E 55, 3851 (1997).

[244] E.P. Gyftopoulos, Maxwell's demon. (I) A thermodynamic exorcism, Physica A 307, 405-420 (2002).

[245] R. Luzzi, A.R. Vasconcellos, and J.G. Ramos, Nonequilibrium statistical mechanics of complex systems: an overview, Rivista Nuovo Cimento, 30(3), 95-158 (2007).

[246] S. Hawling and D. Ben Avraham, Adv. Phys. 36, 695 (1987).

[247] J.R. Dutcher and A.G. Marangoni, Soft Materials (Marcel Dekker, New York, USA, 2005).

[248] A.R. Vasconcellos, E. Laureto, E.A. Meneses, and R. Luzzi, Chaos, Solitons and Fractals 28, 8 (2006).

[249] J.G. Ramos, A. Gorenstein, M.U. Kleinke, T.G. Souza Cruz, and R. Luzzi, J. Mod. Phys. B, 20, 4121 (2006).

[250] P.N. Prasad, J.E. Mark, and T.J. Fai, Editors, Polymers and Other Advanced Materials: Emerging Technologies and Bussiness Oportunities (Plenum, New York, USA, 1996).

[251] W.R. Salaneck, K. Seki, A. Kahn, and J.J. Pireaux, Editors, Conjugated Polymers and Molecular Interfaces: Science and Technology for Photonic and Optoelectronic Applications (Marcel Dekker, New York, USA, 2001).

[252] M.J. Bowden and S.R. Turner, Editors, Polymers for High Technology: Electronics and Photonics (Am. Chem. Soc., Washington, USA, 1987).

[253] C.P. Wong, Polymers for Electronics and Photonic Applications (Academic, San Diego, USA, 1993).

[254] E. Reichmanis and J.H. O'Donnell, Editors, The Effects of Radiation on High-Technology Polymers (Am. Chem. Soc., Washington, USA, 1989).

[255] Y. Lu, Solitons and Polarons in Conducting Polymers (World Scientific, Singapore, 1988).

[256] A.J. Heeger, S. Kivelson, J.R. Schrieffer, and W.P. Su, Solitons in conducting polymers, Rev. Mod. Phys. 60, 781 (1988).

[257] T. Anderson and S. Roth, Braz. J. Phys. 24, 746 (1994).

[258] D. de Kee and K.F. Wissbrum, Polymer Rheology, Physics Today, 51(6), 24-29 (1998).

[259] J.P. Bouchaud and A. Georges, Anomalous diffusion in disordered media: statistical mechanisms, models and physical applications, Phys. Rep. 195(4-5), pp. 127-293 (1990).

[260] S.L. Keller, P. Boltenhagen, D.J. Pine, and J.A. Zasadzinski, Direct observation of shear-induced structures in wormlike micellar solutions by freeze-fracture electron microscopy, Phys. Rev. Lett. 80, 2725 (1998).

[261] S.P. Sullivan et al., J. Non-Newtonian Fluid Mech. 143, 59 (2007); a note in: Editor's choice Science 316, 955 (2007).

[262] Yu. L. Klimontovich, Theor. Math. Phys. 92, 909 (1993).

[263] A. Compte and D. Jou, J. Phys. A: Math. Gen. 29, 4321 (1996).

[264] A. Compte, D. Jou and Y. Katayama, J. Phys. A: Math. Gen. 30, 1023 (1997). 